\title{
Two-sided bounds on minimum-error quantum measurement, on the reversibility of quantum dynamics, and on maximum overlap using directional iterates
}

\author{
Jon Tyson* \\ Jefferson Lab, Harvard University \\ Posted July 10, 2009 \\ Revised June 1, 2010
}

\begin{abstract}
In a unified framework, we estimate the following quantities of interest in quantum information theory:

1. The minimum-error distinguishability of arbitrary ensembles of mixed quantum states.

2. The approximate reversibility of quantum dynamics in terms of entanglement fidelity. (This is referred to as "channel-adapted quantum error recovery" when applied to the composition of an encoding operation and a noise channel.)

3. The maximum overlap between a bipartite pure quantum state and a bipartite mixed state that may be achieved by applying a local quantum operation to one part of the mixed state.
\end{abstract}

4. The conditional min-entropy of bipartite quantum states.

A refined version of the author's techniques [J. Math. Phys. 50, 032016] for bounding the first quantity is employed to give two-sided estimates of the remaining three quantities.

We obtain a closed-form approximate reversal channel. Using a state-dependent Kraus decomposition, our reversal may be interpreted as a quadratically-weighted version of that of Barnum and Knill [J. Math. Phys. 43, 2097]. The relationship between our reversal and Barnum and Knill's is therefore similar to the relationship between Holevo's asymptoticallyoptimal measurement [Theor. Probab. Appl. 23, 411] and the "pretty good" measurement of Belavkin [Stochastics 1, 315] and Hausladen \& Wootters [J. Mod. Optic. 41, 2385]. In particular, we obtain relatively simple reversibility estimates without negative matrix powers at no cost in tightness of our bounds. Our recovery operation is found to significantly outperform the so-called "transpose channel" in the simple case of depolarizing noise acting on half of a maximally-entangled state. Furthermore, our overlap results allow the entangled input state and the output target state to differ, thus obtaining estimates in a somewhat more general setting.

Using a result of König, Renner, and Schaffner [IEEE. Trans. Inf. Th. 55, 4337], our maximum overlap estimate is used to bound the conditional min-entropy of arbitrary bipartite states.

Our primary tool is "small angle" initialization of an abstract generalization of the iterative schemes of Ježek-Řeháček-Fiurášek [Phys. Rev. A 65, 060301], Ježek-Fiurášek-Hradil [Phys. Rev. A 68, 012305], and Reimpell-Werner [Phys. Rev. Lett. 94, 080501]. The monotonicity result of Reimpell [Ph.D. Thesis, 2007] follows in greater generality.

*jonetyson@X.Y.Z, where $\mathrm{X}=$ post, $\mathrm{Y}=$ Harvard, $\mathrm{Z}=$ edu 


\section{Contents}

1 Introduction 3

1.1 Minimum-error detection $\ldots \ldots \ldots \ldots \ldots \ldots$

1.1.1 The relationship to "worst-case" detection . . . . . . . . . . . . . . . . . 4

1.2 Channel-adapted quantum error recovery . . . . . . . . . . . . . . . . 4

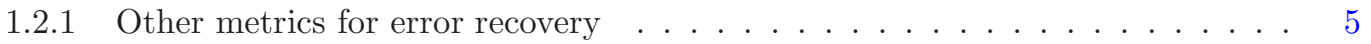

1.3 Quantum conditional min- and max-entropy . . . . . . . . . . . . . . 5

1.4 Directional Iterates: An abstract approach for deriving estimates . . . . . . . . . . 6

1.4.1 Example 1: Ježek-Řeháček-Fiurášek iteration for POVMs . . . . . . . . . . 8

1.4.2 Example 2: Ježek-Fiurášek-Hradil and Reimpell-Werner iterates . . . . . . . 8

1.5 Relevant existing bounds, suboptimal measurements, and approximate reversals . . . 9

1.5.1 Quadratic measurements and Generalized Holevo-Curlander bounds . . . . . 9

1.5.2 The "pretty good" measurement and Barnum \& Knill's distinguishability bound 10

1.5.3 Barnum and Knill's approximate reversal map . . . . . . . . . . . . . . . . . 10

1.5.4 The bounds of Bény and Oreshkov . . . . . . . . . . . . . . . . . 11

1.6 Results . . . . . . . . . . . . . . . . . . . . . . . . . . 12

2 Notation, conventions, and mathematical background $\quad 12$

2.1 Basis-free constructions using dual spaces and double kets . . . . . . . . . . . . . . 14

3 Minimum-error distinction as a maximal seminorm problem $\quad 16$

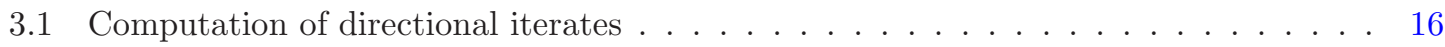

3.2 A "small-angle" guess . . . . . . . . . . . . . . . . . . . . . . 17

3.3 A simple proof of the generalized Holevo-Curlander bounds . . . . . . . . . . . . 18

4 Maximum overlap as a maximal-seminorm problem $\quad 18$

4.1 Computation of directional iterates . . . . . . . . . . . . . . . . . 18

4.2 The restricted maximum-overlap problem . . . . . . . . . . . . . . . . . . 19

4.2 .1 A minor simplification . . . . . . . . . . . . . . . . . . . . 19

$4.2 .2 \quad$ A "small angle" guess . . . . . . . . . . . . . . . . . . . . . . . . . 20

4.2 .3 Angle Estimates . . . . . . . . . . . . . . . . . . . . . 21

4.2 .4 The "Quadratic Overlapper" . . . . . . . . . . . . . . . . . . 23

4.2.5 Estimates for the restricted maximum overlap problem . . . . . . . . . . . 23

4.3 Estimates for quantum conditional min-entropy . . . . . . . . . . . . . . . . . 24

5 Approximate Channel Reversals 25

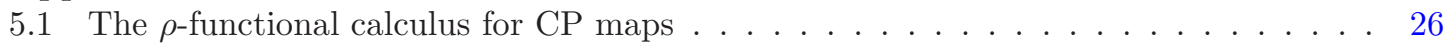

5.2 Quadratic quantum error recovery . . . . . . . . . . . . . . . . . 28

5.3 The relationship between the Quadratic Recovery and Barnum and Knill's reversal 30

5.3.1 Depolarizing noise and the quadratic transpose channel . . . . . . . . . . . . 31

6 Conclusion and future directions $\quad 31$

Appendix A: Canonical Stinespring dilations 32

Appendix B: Reimpell-Werner iteration as directional iteration 33

Appendix C: The relationship between overlap bounds and state distinguishability 34 


\section{Introduction}

This paper considers the following problem of relevance in quantum information theory:

The maximum overlap problem: Let $\mu_{\mathcal{K} \mathcal{H}}$ be a positive semidefinite trace-class operator on $\mathcal{K} \otimes \mathcal{H}$, and let $M_{\mathcal{L H}}$ be positive semidefinite bounded operator on $\mathcal{L} \otimes \mathcal{H}$, where $\mathcal{H}, \mathcal{K}$, and $\mathcal{L}$ are separable Hilbert spaces. What is maximum overlap

$$
\operatorname{MO}\left(\mu_{\mathcal{K} \mathcal{H}}, M_{\mathcal{L H}}\right)=\sup _{\mathcal{R}} \operatorname{Tr}_{\mathcal{L} \mathcal{H}}\left(M_{\mathcal{L H}} \mathcal{R}_{\mathcal{K} \rightarrow \mathcal{L}}\left(\mu_{\mathcal{K} \mathcal{H}}\right)\right),
$$

where the supremum is over all quantum operations $\mathcal{R}$ from $\mathcal{K}$ to $\mathcal{L}$ ?

The maximum-overlap problem has the following important special cases:

\section{The minimum-error quantum detection problem [1-4]:}

If an unknown quantum state $\rho_{k}$ is randomly selected from given ensemble of such states, with what probability may the value of $k$ be determined by a carefully-chosen quantum measurement?

\section{Approximate reversal of quantum dynamics [5-20]:}

Suppose that an arbitrary quantum operation $\mathcal{A}$ acts on a given quantum state $\rho$. How well may the action of $\mathcal{A}$ be reversed by application of a recovery channel $\mathcal{R}$, so as to preserve the entanglement of the original system with the environment? This problem is one of "channel-adapted quantum error recovery" when the operation $\mathcal{A}$ is of the form $\mathcal{A}=\mathcal{N} \circ \mathcal{E}$, where $\mathcal{E}$ is an encoding operation designed to protect against a known noise process $\mathcal{N}$.

\section{Estimation of conditional min-/max-entropy of bipartite quantum states [21]:}

Let $\rho_{A B}$ be a bipartite quantum mixed state. Estimate the conditional min-entropy $H_{\text {min }}(A \mid B)$ of $A$ given $B$.

Since all of these problems are believed to defy closed-form solution, the purpose of this paper is to provide estimates. In section 3 a refined proof of the two-sided "generalized Holevo-Curlander" bounds of $[22,23]$ for case 1 is given. This method is extended in sections 4 and 5 to yield simple two-sided estimates for cases 2-3 and for $\mathrm{MO}(\mu, M)$ in the case of rank-1 $M$.

We briefly introduce each of the cases 1-3 before outlining our approach and surveying closelyrelated work.

\subsection{Minimum-error detection}

The minimum-error quantum detection problem was first studied in the 1960's in connection with the design of optical detectors [24], and it has since become of importance in quantum Shannon theory (for example [25-27]) and in the design of quantum algorithms [28-36]. A generalization to the theory of wave pattern recognition may be found in [37]. Various general upper and/or lower bounds on quantum distinguishability may be found in [14, 22, 23, 25, 32, 38-44].

The minimum-error quantum detection problem is precisely formulated by

Definition 1 Let

$$
\mathcal{E}=\left\{\rho_{k}\right\}_{k \in K}
$$

be an ensemble of quantum states, represented as positive semidefinite operators normalized by a-priori probability, setting

$$
\operatorname{Tr} \rho_{k}=p_{k},
$$


where $p_{k}$ is the likelihood that $\rho_{k}$ will be drawn from $\mathcal{E}$. A quantum measurement [45] is described by a positive-operator-valued measure (POVM), which consists of a vector $M=\left\{M_{k}\right\}_{k \in K}$ of positive semidefinite operators satisfying $\sum M_{k} \leq \mathbb{1}^{[105]}$ (Throughout this paper the operator inequality $A \leq B$ means $B-A$ is positive semidefinite.) The probability that the value $k$ is measured when $M$ is applied to a unit-trace density matrix $\rho$ is given by

$$
\operatorname{Pr}_{M}(k \mid \rho)=\operatorname{Tr} M_{k} \rho .
$$

The success rate for the POVM $M$ to correctly determine the value of $k$ corresponding to a random element of the ensemble $\mathcal{E}$ is given by

$$
P_{\text {succ }}(M)=\sum_{k} p_{k} \operatorname{Pr}_{M}\left(k \mid \frac{\rho_{k}}{p_{k}}\right)=\operatorname{Tr} \sum_{k \in K} M_{k} \rho_{k} .
$$

The minimum-error measurement problem consists of finding a POVM maximizing (4).

\subsubsection{The relationship to "worst-case" detection}

Sometimes one is interested in the "worst-case" distinguishability

$$
\max _{M} \min _{k} \operatorname{Tr} \sum M_{k} \hat{\rho}_{k}
$$

of a collection of unit-trace states $\hat{\rho}_{k}$. As pointed out in [46], the minimax theorem [47] implies that

$$
\max _{M} \min _{k} \operatorname{Tr}\left(M_{k} \hat{\rho}_{k}\right)=\max _{M} \min _{\left\{p_{k}\right\}} \operatorname{Tr} \sum M_{k} p_{k} \hat{\rho}_{k}=\min _{\left\{p_{k}\right\}} \max _{M} \operatorname{Tr} \sum M_{k} p_{k} \hat{\rho}_{k},
$$

where $\left\{p_{k}\right\}$ represents a probability distribution. In particular, single-instance bounds (for fixed $\left.\left\{p_{k}\right\}\right)$ may in principle be minimized over all distributions $\left\{p_{k}\right\}$ to give corresponding "worst-case" bounds.

\subsection{Channel-adapted quantum error recovery}

The following problem is of importance in quantum information theory, quantum communication, and quantum computing:

Suppose that one wishes to store, process, or transmit quantum data using a process that is subject to noise or loss. How well may the effects of this noise be avoided, corrected, or eliminated by encoding the data into a protected form, from which it may be later recovered unharmed by this noise?

This problem arises in any physical implementation of quantum communication or computation, since unmitigated interactions with the environment tend to corrupt quantum signals or memory. By the celebrated "threshold theorem" [48-52], one may in principle use error correction and concatenated quantum codes to perform an arbitrary quantum computation in the presence of noise below a fixed "threshold" amount.

Standard quantum error correction seeks to design encoding and decoding maps which exactly correct for a given class of errors. Early successes of this program were the first codes that could protect against arbitrary single-qubit errors [53-55], followed by general theoretical advances of [56], and by the construction of codes that correct for arbitrary single-qubit errors by encoding a single qubit into five $[57,58]$.

Alternatively, one may consider approximate quantum error correction. For example, Leung et al [59] consider relaxed error correction criteria to allow for efficient correction of a known dominant noise process. Furthermore, Crépeau, Gottesman, and Smith [60] construct approximate error correcting codes which asymptotically correct twice as many arbitrary local errors as would 
be possible under exact error correction, even though they achieve fidelity exponentially close to 1 in the limit of long codes.

Under the banner of approximate channel adapted error correction, a number of authors [7-20] alternatively have sought to treat quantum encoding and/or recovery as optimization problems. Mathematically, given a "noise" channel $\mathcal{N}$ one seeks an encoding operation $\xi$ and a recovery operation $\mathcal{R}$ so that the composition

$$
\Xi=\mathcal{R} \circ \mathcal{N} \circ \xi
$$

is as close to the identity channel as possible. Measures of "closeness" to the identity include

Definition 2 Let $\rho$ be a mixed quantum state over a Hilbert space $\mathcal{H}$, which may be represented as a pure quantum state $\left|\psi_{\rho}\right\rangle_{\mathcal{H E}}$ of the original system entangled with an environment $\mathcal{E}$. The entanglement fidelity [61] of the quantum operation $\Xi: B^{1}(\mathcal{H}) \rightarrow B^{1}(\mathcal{H})$ is given by

$$
F_{e}(\rho, \Xi)=\left\langle\psi_{\rho}\left|\Xi\left(\left|\psi_{\rho}\right\rangle\left\langle\psi_{\rho}\right|\right)\right| \psi_{\rho}\right\rangle .
$$

(Note that the choice of purification does not affect the defined quantity.) The channel fidelity is the entanglement fidelity when $\rho$ is taken to be maximally-mixed. Given a collection of states $\rho_{k}$ with a-priori probabilities $p_{k}$, one defines the average entanglement fidelity [14]

$$
\bar{F}_{e}\left(\left\{\left(\rho_{k}, p_{k}\right)\right\}, \Xi\right)=\sum p_{k} F_{e}\left(\rho_{k}, \Xi\right) .
$$

Following $[8,11,14,16]$, we shall fix the encoding operation $\xi$ and the noise process $\mathcal{N}$. In particular, we focus on the problem of finding an approximately optimal quantum recovery map, or channel reversal, for the composed map

$$
\mathcal{A}=\mathcal{N} \circ \xi
$$

in the sense of entanglement fidelity.

\subsubsection{Other metrics for error recovery}

A number of works have considered other measures of reversibility of quantum channels. Kretschmann, Schlingermann, and Werner [62] have obtained two-sided bounds on the CB-norm reversibility of channels in terms of the CB-distance between the complementary channel and a depolarizing channel. Ng and Mandayam [20] have employed the transpose channel (a special case of Barnum and Knill's [14] reversal) to study quantum error correction using the metric of worst-case (nonentanglement) fidelity. Yamamoto, Hara, and Tsumura [17] considered a fixed encoding operation $\mathcal{E}$ and used semidefinite programing to find a sub-optimal channel $\mathcal{R}$ to roughly optimize the "worstcase" entanglement fidelity

$$
\max _{\mathcal{R}} \min _{\rho} F_{e}(\rho, \mathcal{R} \circ \mathcal{N} \circ \mathcal{E})
$$

More will be said about worst-case bounds in section 1.5.4, below.

\subsection{Quantum conditional min- and max-entropy}

The following related quantities (and their $\varepsilon$-smooth counterparts) are of interest in quantum cryptography (for example [21,63-68]) and/or in studies of non-identically distributed and/or nonasymptotic problems in quantum information theory (for example [69-73]):

Definition 3 Let $\rho_{A B}$ be a bipartite density operator on $\mathcal{H}_{A} \otimes \mathcal{H}_{B}$. The min-entropy of $A$ conditioned on $B$ [21, 70] is defined by

$$
H_{\min }(A \mid B)_{\rho}:=-\log _{2} \inf _{v_{B}}\left\{\operatorname{Tr} v_{B} \mid \rho_{A B} \leq \mathbb{1}_{A} \otimes v_{B}\right\},
$$

where the infimum ranges over positive semidefinite $v_{B}$. The max-entropy of $\boldsymbol{A}$ conditioned on $\boldsymbol{B}$ [21, 70] is defined by

$$
H_{\max }(A \mid B)_{\rho}:=-H_{\min }(A \mid C)_{\rho},
$$


where the min-entropy on the RHS is evaluated for a purification $\rho_{A B C}$ of $\rho_{A B}$. The maxinformation [73] that $B$ has about $A$ is given by

$$
I_{\max }(A: B)_{\rho}=H_{\min }(A \mid B)_{\rho_{A}^{-1 / 2} \rho_{A B} \rho_{A}^{-1 / 2}}
$$

where $\rho_{A}=\operatorname{Tr}_{B} \rho_{A B}$.

Estimates of $H_{\min }(A \mid B)_{\rho}$ are obtained as a corollary of our estimates for maximum overlap in conjunction with the following recent theorem:

Theorem 4 (König, Renner, Schaffner [70]) Let the Hilbert spaces $\mathcal{H}_{A}$ and $\mathcal{H}_{B}$ be finite-dimensional. Then the min-entropy of $A$ conditioned on $B$ for the state $\rho_{A B}$ may be expressed as

$$
H_{\text {min }}(A \mid B)_{\rho}=-\log \left(\operatorname{dim}\left(\mathcal{H}_{A}\right) \sup _{\mathcal{R}}\left(\left\langle\Phi_{A A^{\prime}}\left|\mathcal{R}_{B \rightarrow A^{\prime}}\left(\rho_{A B}\right)\right| \Phi_{A A^{\prime}}\right\rangle\right)\right),
$$

where $\Phi_{A A^{\prime}}$ is a bipartite maximally-entangled state between $A$ and reference system $A^{\prime} \simeq A$, and where the supremum is over quantum operations from $B$ to $A^{\prime}$.

\subsection{Directional Iterates: An abstract approach for deriving estimates}

The first step in proving our estimates will be to recast all of the problems of the first section as instances of

Problem 5 (Maximal seminorm problem) Let $S$ be a subset of a real or complex semidefinite inner product space $V$. Find a maximal-seminorm element of $S$. (A semidefinite inner product has all the usual properties of an inner product, except that one may have $\langle x, x\rangle=0$ for nonzero $x$.)

The following generalization of the iterative schemes of $[6,7,10,74]$, will prove useful for analyzing this class of problems:

Definition 6 An abstract Ježek-Řeháček-Fiurášek-Hradil-Reimpell-Werner iterate of $g \in$ $V$ is an element $g^{(+)} \in S$ which maximizes $\operatorname{Re}\left\langle g^{(+)}, g\right\rangle$. Such $g^{(+)}$will also be called directional iterates.

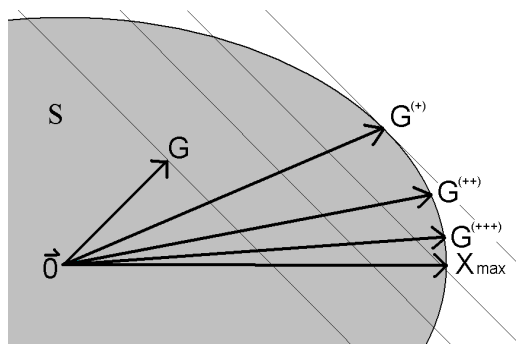

Fig 1a: Iterates converging on $x_{\max }$. (contours drawn orthogonal to $G$.)

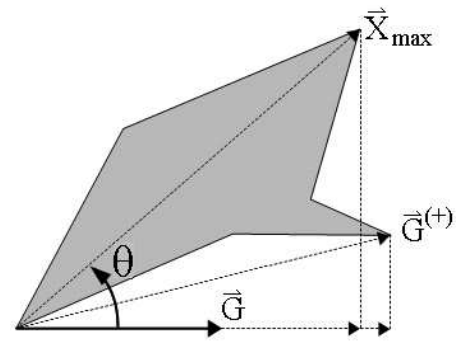

Fig 1b: Note that

$\left\|X_{\max }\right\| \geq\left\|G^{(+)}\right\| \geq\left\|\Pi G^{(+)}\right\| \geq\left\|\Pi X_{\max }\right\|$, with approximate equality for reasonably small $\theta$. Here $\Pi=|G\rangle\langle G|$, and $V$ has real scalars. $A$ complex variant appears as inequality 13 . 
Useful properties of these iterates are given by

Lemma 7 (Geometric properties of directional iterates) Suppose that $S \subseteq V$ has a maximalseminorm vector $x_{\max }$, and assume that each $g \in V$ admits a directional iterate $g^{(+)}$. Then

G1. One has the following inequalities

$$
\left\|x_{\max }\right\| \geq\left\|g^{(+)}\right\| \geq \Lambda(g) \geq\left\|x_{\max }\right\| \cos (\theta)
$$

where

$$
\begin{aligned}
& \Lambda(g):=\operatorname{Re}\left\langle g^{(+)}, \frac{g}{\|g\|}\right\rangle \\
& \cos \theta:=\operatorname{Re} \frac{\left\langle g, x_{\max }\right\rangle}{\|g\|\left\|x_{\max }\right\|}
\end{aligned}
$$

G2. The map $g \mapsto g^{(+)}$is seminorm-increasing on $S$. In particular, if $g \in S$ then

$$
\left\|g^{(+)}\right\|^{2} \geq\|g\|^{2}+\left\|g^{(+)}-g\right\|^{2} .
$$

Note: The importance of property $G 1$ is this: If one can construct a guess $g$ subtending a reasonably small angle with $x_{\max }$ then both $\Lambda(g)$ and $\left\|g^{(+)}\right\|$are reasonably good estimates for $\left\|x_{\max }\right\|$. (Note that although $\left\|g^{(+)}\right\|$is a closer approximation to $\left\|x_{\max }\right\|$, in our applications $\Lambda(g)$ will have a much simpler expression.)

Proof. To prove property $G 1$, note that

$$
\left\|x_{\max }\right\| \geq\left\|g^{(+)}\right\| \geq \frac{\operatorname{Re}\left\langle g^{(+)}, g\right\rangle}{\|g\|} \geq \frac{\operatorname{Re}\left\langle x_{\max }, g\right\rangle}{\|g\|}=\left\|x_{\max }\right\| \cos (\theta) .
$$

The first inequality is trivial, the second is Schwarz's, and the third is by the definition of $g^{(+)}$.

To prove property $G 2$, write

$$
\left\|g^{(+)}\right\|^{2}=\left\|g^{(+)}-g\right\|^{2}+\|g\|^{2}+2 \operatorname{Re}\left(\left\langle g^{(+)}, g\right\rangle-\langle g, g\rangle\right) .
$$

The last term on the RHS is nonnegative by the definition of $g^{(+)}$.

We now may set forth the following:

\section{General strategy for estimating maximal seminorms:}

1. Find a "small angle guess" $g$, such that the angle defined by (15) is provably small in some approximate sense.

2. Obtain two-sided bounds for $\left\|x_{\max }\right\|$ using this bound on $\theta$ in conjunction with (13).

3. Make this bound explicit by computing $g^{(+)}$and $\Lambda(g)$.

By property $G 2$ of Lemma 7, one may have some hope of obtaining a maximal element as the limit of repeated iteration, as occurs in Fig. 1a. In sections 1.4.1-1.4.2 we review numerical schemes in the literature which may be seen as examples of this process. (These sections may be skimmed on first reading.) 


\subsubsection{Example 1: Ježek-గ̌eháček-Fiurášek iteration for POVMs}

Ježek, Řeháček, and Fiurášek (JRF) [74, 75] proposed an unproven numerical method for computing optimal POVMs, ${ }^{[106]}$ using iteration of the mapping $M \mapsto M^{(\oplus)}$ given by

Definition 8 The Ježek-Řeháček-Fiurášek (JRF) iterate of a POVM $M=\left\{M_{k}\right\}_{k \in K}\left[{ }^{7} 4\right.$, 75] is the POVM defined by

$$
M_{k}^{(\oplus)}=\left(\sum_{\ell \in K} \rho_{\ell} M_{\ell} \rho_{\ell}\right)^{-1 / 2^{+}} \rho_{k} M_{k} \rho_{k}\left(\sum_{\ell \in K} \rho_{\ell} M_{\ell} \rho_{\ell}\right)^{-1 / 2^{+}}
$$

Here the negative matrix power is defined by

$$
A^{-s^{+}}=\sum_{\lambda_{j}>0} \lambda_{j}^{-s} \Pi_{j}
$$

for $s \geq 0$ and self-adjoint $A$ with spectral decomposition $A=\sum \lambda_{j} \Pi_{j}$.

Ježek, Řeháček, and Fiurášek made the following:

Numerical Observation 9 (JRF $[\mathbf{7 4}, \mathbf{7 5}]$ ) JRF iteration monotonically increases success rate:

$$
P_{\text {succ }}\left(M^{(\oplus)}\right) \geq P_{\text {succ }}(M) \text {. }
$$

Furthermore, iteration of this map starting from $\left\{M_{k}=\mathbb{1}\right\}$ converges to an optimal measurement

$$
\lim _{j \rightarrow \infty} P_{\text {succ }}\left(M^{(\oplus)^{j}}\right)=P_{\text {succ }}\left(M^{o p t}\right) .
$$

In section 3.1, JRF iteration is exhibited as a disguised form of directional iteration. JRF's numerically-observed monotonicity then follows immediately from property $G 2$ of lemma 7 .

\subsubsection{Example 2: Ježek-Fiurášek-Hradil and Reimpell-Werner iterates}

Ježek, Fiurášek, and Hradil (JFH) [6,75] proposed an unproven numerical scheme for the maximumlikelihood problem [75-78] in quantum process tomography, which contains the maximum-overlap problem (1) as a special case. ${ }^{[107]}$

Reimpell and Werner [7, 10] introduced a mild generalization of this special case of JRH's algorithm, for use in finding maximizers of the following:

Definition 10 A Reimpell-Werner functional [7, 10] $\mathcal{R} \mapsto f(\mathcal{R})$ is a linear functional of linear transformations $\mathcal{R}: B^{1}(\mathcal{K}) \rightarrow B^{1}(\mathcal{L})$ such that $f(\mathcal{R}) \geq 0$ for all completely positive $\mathcal{R}$.

Reimpell and Werner were interested in the special cases of approximate quantum error recovery and quantum encoding in the sense of channel fidelity. In particular, setting

$$
f_{\mathcal{N}}(\mathcal{E}, \mathcal{R})=F_{e}(\mathbb{1} / \operatorname{dim} \mathcal{H}, \mathcal{R} \circ \mathcal{N} \circ \mathcal{E})
$$

where $\mathcal{N}$ is a known noise map, they alternatively optimized the encoder $\mathcal{E}$ and decoder $\mathcal{R}$ in a seesaw fashion.

By analogy with the matrix-power method [7, 10], they proposed an unproven numerical method for maximizing $f(\mathcal{R})$ by iteration of the following map:

Definition 11 Let $\mathcal{L}$ and $\mathcal{K}$ be finite-dimensional, and represent the Reimpell-Werner functional $f$ as

$$
f(\mathcal{R})=\operatorname{Tr}_{\mathcal{L} \mathcal{K}^{*}}(F \tilde{\mathcal{R}})
$$


where $\tilde{\mathcal{R}} \in B^{1}\left(\mathcal{L} \otimes \mathcal{K}^{*}\right)$ is the Choi matrix of $\mathcal{R}$ (see Definition 27) and $F$ is a positive operator on $\mathcal{L K}^{*}$. The Reimpell-Werner iterate $\mathcal{R}^{\oplus}$ of $\mathcal{R}$ [7, 10] is the quantum operation with Choi matrix

$$
\widetilde{\mathcal{R}}^{\oplus}=\Gamma^{-1 / 2^{+}} F \tilde{R} F \Gamma^{-1 / 2^{+}},
$$

where $\Gamma: \mathcal{L K}^{*} \rightarrow \mathcal{L K}^{*}$ is given by

$$
\Gamma=\mathbb{1}_{\mathcal{L}} \otimes \underset{\mathcal{L}}{\operatorname{Tr}}(F \tilde{R} F)
$$

Reimpell [10] proved the monotonicity property $f\left(\mathcal{R}^{\oplus}\right) \geq f(\mathcal{R})$ using a clever matrix analysis argument. In particular, the optimal map $\mathcal{R}$ is a fixed point of this iteration.

In Appendix B we show that Reimpell-Werner iteration (and the special case of restricted JRH iteration) may be viewed as directional iteration on the corresponding space of Stinespring dilations. In particular, Reimpell's monotonicity result is exhibited as a special case of Lemma 7.

\subsection{Relevant existing bounds, suboptimal measurements, and approxi- mate reversals}

\subsubsection{Quadratic measurements and Generalized Holevo-Curlander bounds}

Definition 12 Let $\mathcal{E}=\left\{p_{k}\left|\psi_{k}\right\rangle\left\langle\psi_{k}\right|\right\}_{k \in K}$ be an ensemble of pure states. Then Holevo's pure state measurement [43] is given by $M_{k}=\left|e_{k}\right\rangle\left\langle e_{k}\right|$, where

$$
e_{k}=e_{k}^{\text {Holevo }}:=\left(\sum p_{k}^{2}\left|\psi_{k}\right\rangle\left\langle\psi_{k}\right|\right)^{-1 / 2^{+}} p_{k} \psi_{k} .
$$

Holevo constructed this measurement using an approximate minimal principle, and proved

Theorem 13 (Holevo's asymptotic optimality theorem [43]) Holevo's measurement is asymptotically-optimal for distinguishing pure states in the sense that for fixed probabilities $\left\{p_{k}\right\}$ one has

$$
\frac{P_{\text {fail }}\left(\left\{e_{k}^{\text {Holevo }}\right\}\right)}{P_{\text {fail }}^{\text {optimal }}} \rightarrow 1
$$

as the $\psi_{k}$ are varied so that $\left\langle\psi_{i}, \psi_{j}\right\rangle \rightarrow \delta_{i j}$. Here $P_{\text {fail }}=1-P_{\text {succ }}$ represents the failure rate.

A natural mixed-state generalization of Holevo's measurement is given by

Definition 14 The quadratically-weighted measurement [22, 74] for distinguishing the ensemble (2) is the first Ježek- Řeháček-Fiurášek iterate

$$
M_{k}^{Q W}=\left(\sum_{\ell} \rho_{\ell}^{2}\right)^{-1 / 2^{+}} \rho_{k}^{2}\left(\sum_{\ell} \rho_{\ell}^{2}\right)^{-1 / 2^{+}} .
$$

Remark: The quadratically-weighted measurement is an example of a Belavkin-Maslov measurement (see page 39 of [37]).

Generalizing the pure-state results of Holevo [43] and Curlander [44], the author proved the following:

Theorem 15 (Generalized Holevo-Curlander bounds [22]) One has the following bounds on the success rate of the optimal measurement $M^{\text {opt }}$ for distinguishing the ensemble $\mathcal{E}$ of Definition 1:

$$
\Lambda^{2} \leq P_{\text {succ }}\left(M^{Q W}\right) \leq P_{\text {succ }}\left(M^{o p t}\right) \leq \Lambda,
$$

where

$$
\Lambda=\operatorname{Tr} \sqrt{\sum \rho_{k}^{2}} \leq 1
$$

Note: The upper bound of (27) was essentially a special case of a pre-existing bound of Ogawa and Nagaoka [23], which is a simple consequence of matrix monotonicity. 


\subsubsection{The "pretty good" measurement and Barnum \& Knill's distinguishability bound}

Another approximately-optimal measurement is the linearly-weighted measurement given by

Definition 16 The Belavkin-Hausladen-Wootters "pretty good" measurement (PGM) [79-82] is given by

$$
M_{k}^{P G M}=\left(\sum \rho_{\ell}\right)^{-1 / 2^{+}} \rho_{k}\left(\sum \rho_{\ell}\right)^{-1 / 2^{+}}
$$

A comparison of the PGM with Holevo's pure state measurement was conducted in [83]. It was found that Holevo's measurement outperforms the PGM for ensembles of two pure states, and that the PGM does NOT satisfy Holevo's asymptotic optimality property (25).

The PGM is approximately-optimal for "reasonably-distinguishable" ensembles in the following precise sense:

Theorem 17 (Barnum-Knill [14]) The success rate of the PGM satisfies

$$
\frac{P_{\text {succ }}\left(M^{P G M}\right)}{P_{\text {succ }}\left(M^{o p t}\right)} \geq P_{\text {succ }}\left(M^{o p t}\right) \text {, }
$$

where $M^{o p t}$ is an optimal measurement.

Re-expressing this inequality in terms of $P_{\text {fail }}=1-P_{\text {succ }}$, one sees that the PGM has a failure rate within a factor of two of the optimal:

$$
P_{\text {fail }}\left(M^{\mathrm{opt}}\right) \leq P_{\text {fail }}\left(M^{\mathrm{PGM}}\right) \leq\left(1+P_{\text {succ }}\left(M^{\mathrm{opt}}\right)\right) P_{\text {fail }}\left(M^{\mathrm{opt}}\right) \leq 2 \times P_{\text {fail }}\left(M^{\mathrm{opt}}\right)
$$

The relationship between Barnum and Knill's bound (30) and the bounds of Theorem 15 is explained by the following proposition:

Proposition 18 (Comparison with the Barnum-Knill bounds) Both of the lower bounds of inequality 27 are sufficiently tight to also satisfy Barnum and Knill's tightness relation (30):

$$
\frac{P_{\text {succ }}\left(M^{Q W}\right)}{P_{\text {succ }}\left(M^{o p t}\right)} \geq \frac{\Lambda^{2}}{P_{\text {succ }}\left(M^{o p t}\right)} \geq P_{\text {succ }}\left(M^{o p t}\right) .
$$

In particular, $P_{\text {fail }}\left(M^{Q W}\right), 2(1-\Lambda)$, and $1-\Lambda^{2}$ all lie in the interval $\left[P_{\text {fail }}\left(M^{o p t}\right), 2 \times P_{\text {fail }}\left(M^{o p t}\right)\right]$. Proof. Equation 32 follows immediately by double application of inequality 27. The claimed inclusions follow as in inequality 31 , where one additionally uses the inequality $1-\Lambda^{2} \leq 2(1-\Lambda)$.

\subsubsection{Barnum and Knill's approximate reversal map}

Generalizing the "pretty good" measurement ${ }^{[108]}$, Barnum and Knill have constructed a reversal of an arbitrary quantum operation $\mathcal{A}: B^{1}(\mathcal{H}) \rightarrow B^{1}(\mathcal{K})$ that is approximately optimal for reasonably reversible $\mathcal{A}$ in a precise sense:

Theorem 19 (Barnum-Knill [14]) Assume that the density operators $\rho_{k} \in B^{1}(\mathcal{H})$ of equation 8 commute, set $\rho=\sum p_{k} \rho_{k}$, and let $\mathcal{A}^{\dagger}: B(\mathcal{K}) \rightarrow B(\mathcal{H})$ be the adjoint of $\mathcal{A}$ (see Def. 24, below). Then the recovery operation

$$
\mathcal{R}^{B K}(v)=\sqrt{\rho} \mathcal{A}^{\dagger}\left((\mathcal{A}(\rho))^{-1 / 2^{+}} v(\mathcal{A}(\rho))^{-1 / 2^{+}}\right) \sqrt{\rho}
$$

is approximately optimal in the sense that

$$
\frac{\bar{F}_{e}\left(\left\{\rho_{k}, p_{k}\right\}, \mathcal{R}^{B K} \circ \mathcal{N}\right)}{\max _{\mathcal{R}} \bar{F}_{e}\left(\left\{\rho_{k}, p_{k}\right\}, \mathcal{R} \circ \mathcal{N}\right)} \geq \max _{\mathcal{R}} \bar{F}_{e}\left(\left\{\rho_{k}, p_{k}\right\}, \mathcal{R} \circ \mathcal{N}\right)
$$

where $\bar{F}_{e}$ is the average entanglement fidelity of equation 8. 
A special case of eq. 33 is of recent [20] interest in the literature:

Definition 20 The transpose channel [84] is the special case of the Barnum-Knill reversal $\mathcal{R}^{B K}$ for maximally-mixed $\rho$.

A reversal of approximately optimal entanglement fidelity which is closely related to the quadratic measurement will be constructed in section 5 .

\subsubsection{The bounds of Bény and Oreshkov}

Generalizing the problem of quantum error-recovery, Bény and Oreshkov [18] have more-generally considered channel simulation. In particular, they consider the "worst-case" entanglement fidelity

$$
\max _{\mathcal{R}} \min _{\rho} F_{\rho}(\mathcal{R} \mathcal{A}, \mathcal{M})
$$

with which the channel $\mathcal{A}$ may be used to simulate the channel $\mathcal{M}$. Here one has

$$
F_{\rho}(\mathcal{N}, \mathcal{M})=\min _{\rho} f\left(\mathcal{N}_{\mathcal{H} \rightarrow \mathcal{K}}\left(\left|\psi_{\rho}\right\rangle_{\mathcal{H} \mathcal{H}_{R}}\left\langle\psi_{\rho}\right|\right), \mathcal{M}_{\mathcal{H} \rightarrow \mathcal{K}}\left(\left|\psi_{\rho}\right\rangle_{\mathcal{H} \mathcal{H}_{R}}\left\langle\psi_{\rho}\right|\right)\right)
$$

where $\psi_{\rho}$ is a purification of $\rho$ and (changing their conventions slightly) $f(\rho, \sigma)=(\operatorname{Tr} \sqrt{\sqrt{\rho} \sigma \sqrt{\rho}})^{2}$ is the fidelity between the states $\rho$ and $\sigma$. Note that quantum error recovery is the $\mathcal{M}=\mathbb{1}$ special case. Employing the min-max Theorem and a beautiful (and short!) duality argument involving complementary channels, they obtain the following theorem:

Theorem 21 (Bény-Oreshkov [18]) One has the worst-case recovery bounds

$$
\left(\frac{3}{4}+\frac{1}{4} \tilde{\Lambda}_{\sigma}\right)^{2} \geq \max _{\mathcal{R}} \min _{\rho} \mathcal{F}_{e}(\rho, \mathcal{R} \circ \mathcal{A}) \geq \tilde{\Lambda}_{\sigma}^{2}
$$

where the state $\sigma$ is an adjustable parameter, $\mathcal{A}$ has Kraus decomposition $\mathcal{A}(\mu)=\sum E_{i} \mu E_{i}^{\dagger}$, and

$$
\tilde{\Lambda}_{\sigma}:=\min _{\rho} \operatorname{Tr} \sqrt{\sum E_{i} \rho^{2} E_{j}^{\dagger} \times \operatorname{Tr}\left(E_{j} \sigma E_{i}^{\dagger}\right)} .
$$

Furthermore, if $\rho$ is fixed then one obtains

$$
\left(\frac{3}{4}+\frac{1}{4} F_{\rho}(\hat{\mathcal{A}}, S)\right)^{2} \geq \max _{\mathcal{R}} F_{e}(\rho, \mathcal{R} \circ \mathcal{A}) \geq\left(F_{\rho}(\hat{\mathcal{A}}, S)\right)^{2}
$$

where $\hat{\mathcal{A}}$ is a channel complementary to $\mathcal{A}$ and $S(\sigma)=\hat{\mathcal{A}}(\rho) \times \operatorname{Tr} \sigma$.

\section{Remarks:}

1. There is an apparent, but unexplained, relationship between our work below and the results of Bény-Oreshkov, which appeared in arXiv preprint form almost-simultaneously to ours. Further will be said on this matter in [19]. (See Theorem 44 and Proposition 45, below.)

2. It is important to note that in the finite-dimensional case that one has the identity

$$
\max _{\mathcal{R}} \min _{\rho} F_{e}(\rho, \mathcal{R} \circ \mathcal{N} \circ \mathcal{E})=\min _{\rho} \max _{\mathcal{R}} F_{e}(\rho, \mathcal{R} \circ \mathcal{N} \circ \mathcal{E})
$$

This follows from the min-max Theorem [85], where the convexity of the mapping $\rho \mapsto$ $F_{e}(\rho, \mathcal{R} \circ \mathcal{N} \circ \mathcal{E})$ is evident from equation 1.10 of [15] and where one may take the recovery $\mathcal{R}$ to range over the convex set of quantum operations (trace non-increasing completely positive maps). In particular, one may obtain "worst-case" recovery bounds (albeit with unevaluated minimization over $\rho$ ) from single-instance bounds on $F_{e}(\rho, \mathcal{R} \circ \mathcal{N})$, which we exclusively consider below. 


\subsection{Results}

Section 1.4 has already introduced directional iteration as an abstract method for estimating solutions of maximal-seminorm problems. This incorporates several explicitly-defined numerical iterative schemes, including:

- The iteration of Ježek, Řeháček, and Fiurášek for computing optimal quantum measurements.

- The iteration of M. Ježek, J. Fiurášek, and Z. Hradil as restricted to the maximum-overlap problem.

- The iteration of Reimpell and Werner for numerically optimizing quantum error correction (both encoding and recovery).

Defined by a minimal-principle, directional iteration monotonically increases seminorm essentially by construction. In particular:

- Ježek, Řeháček, and Fiurášek's numerical observation that their iteration only increases success rate is proven in greater generality.

- This gives a short proof of Reimpell's monotonicity Theorem (pp. 39-42 of [10]) for iterative optimization of quantum error correction.

Section 3 introduces our techniques by presenting a new proof of the generalized HolevoCurlander bounds (Theorem 15) on the distinguishability of arbitrary ensembles of mixed quantum states.

In section 4, Theorem 39 gives concise two-sided bounds for the maximum overlap problem (1), in the restricted case that $M_{\mathcal{L H}}$ is rank 1. Corollary 40 bounds the quantum conditional minentropy. Appendix $\mathrm{C}$ shows how one may apply these bounds to recover the bounds of section 3.

Theorem 44 of section 5 applies our overlap bounds to estimate approximate channel reversibility in the sense of entanglement fidelity. (The bounds of section 4.2.5 more generally allow the entangled input and output states to differ, however.) Our channel-reversibility estimates apply to the case of channel-adapted approximate quantum error recovery.

Section 5.3 compares our reversibility estimates and approximate reversal map to those of Barnum and Knill. Although our bounds take a particularly simple form, they are still sufficiently accurate to satisfy the tightness relation (34) satisfied by the bounds of Barnum and Knill. The relationship between our recovery map and Barnum and Knill's is found to be analogous to the relationship between Holevo's asymptotically optimal measurement and the so-called "pretty good" measurement. Furthermore, our recovery operation is found to significantly outperform the transpose channel in the case of depolarizing noise acting on half of a maximally-entangled state.

The conclusion points out directions for future research.

\section{Notation, conventions, and mathematical background}

The reader who is only interested in minimum-error distinguishability bounds should proceed directly to section 3 , referring back only as directed.

Definition 22 Let $\mathcal{H}$ and $\mathcal{K}$ be Hilbert spaces, and let $A: \mathcal{H} \rightarrow \mathcal{K}$ be a bounded linear operator. The absolute value is $|A|=\sqrt{A^{\dagger} A}$. The space $B^{1}(\mathcal{H} \rightarrow \mathcal{K})$ consists of all operators of finite trace norm $\|A\|_{1}:=\operatorname{Tr}|A|$. The space $B^{2}(\mathcal{H} \rightarrow \mathcal{K})$ consists of all operators of finite Hilbert-Schmidt norm $\|A\|_{2}:=\sqrt{\operatorname{Tr} A^{\dagger} A}$. This space has the inner product

$$
\langle A, B\rangle=\operatorname{Tr} A^{\dagger} B .
$$


The space $B(\mathcal{H} \rightarrow \mathcal{K})$ consists of all operators of finite operator norm, given by

$$
\|A\|=\|A\|_{\infty}=\sup _{0 \neq \psi \in \mathcal{H}} \frac{\|A \psi\|}{\|\psi\|} .
$$

When $\mathcal{H}=\mathcal{K}$, these spaces will be denoted by $B(\mathcal{H}), B^{1}(\mathcal{H})$, and $B^{2}(\mathcal{H})$, for short. An operator $A$ is a contraction if $\|A\| \leq 1$.

It is assumed that the reader is familiar with the following trace-norm inequalities, which may be found in [86]:

$$
\begin{aligned}
|\operatorname{Tr} A| & \leq\|A\|_{1}=\left\|A^{\dagger}\right\|_{1} \text { if } \mathcal{K}=\mathcal{H} \\
\|W A\|_{1} & \leq\|W\|_{\infty} \times\|A\|_{1}
\end{aligned}
$$

Furthermore,

$$
\sup _{\|U\| \leq 1} \operatorname{Re}\left(\operatorname{Tr} A^{\dagger} U\right)=\|A\|_{1},
$$

where $A: \mathcal{H} \rightarrow \mathcal{K}$ and the supremum is over contractions $U: \mathcal{H} \rightarrow \mathcal{K}$. It follows simply from the singular value decomposition that $U$ is a maximizer of (43) iff

$$
\left.U\right|_{\operatorname{Ran}\left(A^{\dagger} A\right)}=A\left(A^{\dagger} A\right)^{-1 / 2^{+}}
$$

where $\left(A^{\dagger} A\right)^{-1 / 2^{+}}$is defined by (18).

Definition 23 Let $A$ be a self-adjoint operator. The positive projection $\Pi_{+}(A)$ is the projection onto the closure of the range of the positive part of $A$. In particular, if $A$ has spectral decomposition $A=\sum \lambda_{i}\left|\psi_{i}\right\rangle\left\langle\psi_{i}\right|$ then

$$
\Pi_{+}(A)=\sum_{\lambda_{i}>0}\left|\psi_{i}\right\rangle\left\langle\psi_{i}\right|
$$

A more thorough discussion of most of the following terms may be found in [45]:

Definition 24 A quantum state is a trace-class positive semidefinite operator $\rho$ on a Hilbert space $\mathcal{H}$. (Generally states are of unit trace, although in section 3 it will be convenient to normalize them by a-priori probability.) The support $\operatorname{supp}(A)$ of the transformation $A: \mathcal{H} \rightarrow \mathcal{K}$ is the closure of the range of $A^{\dagger} A$, or equivalently the orthogonal complement of the null-space of $A$. $A$ quantum channel is a trace preserving completely positive map. A quantum operation is a trace non-increasing completely positive map. A linear operator $U: \mathcal{K} \rightarrow \mathcal{L} \otimes \mathcal{E}$ is a Stinespring dilation [87] of a completely positive map $\mathcal{R}: B^{1}(\mathcal{K}) \rightarrow B^{1}(\mathcal{L})$ if

$$
\mathcal{R}(\rho)=\operatorname{Tr}_{\mathcal{E}}\left(U \rho U^{\dagger}\right)
$$

for all $\rho \in B^{1}(\mathcal{K})$. The adjoint $\mathcal{R}^{\dagger}: B(\mathcal{L}) \rightarrow B(\mathcal{K})$ has the defining property that

$$
\operatorname{Tr}_{\mathcal{L}}\left(X_{\mathcal{L}} \mathcal{R}_{\mathcal{K} \rightarrow \mathcal{L}}\left(Y_{\mathcal{K}}\right)\right)=\operatorname{Tr}_{\mathcal{K}}\left(\mathcal{R}_{\mathcal{L} \rightarrow \mathcal{K}}^{\dagger}\left(X_{\mathcal{L}}\right) Y_{\mathcal{K}}\right)
$$

for $X \in B(\mathcal{L})$ and $Y \in B^{1}(\mathcal{K})$.

It is important to note that if $\mathcal{R}$ and $U$ are related by (46) then $\mathcal{R}$ is a channel iff $U$ is an isometry $\left(U^{\dagger} U=\mathbb{1}\right)$, and $\mathcal{R}: B^{1}(\mathcal{K}) \rightarrow B^{1}(\mathcal{L})$ is a quantum operation iff $U$ is a contraction. Furthermore, it is observed in Appendix A that each quantum operation $\mathcal{A}$ has a canonical dilation with the canonical environment

$$
\mathcal{E}=\mathcal{L}_{\mathcal{E}}^{*} \otimes \mathcal{K}_{\mathcal{E}}
$$


where $\mathcal{K}_{\mathcal{E}}$ and $\mathcal{L}_{\mathcal{E}}^{*}$ are copies of $\mathcal{K}$ and the dual space of $\mathcal{L}$, respectively.

Tensor product notation: A linear operator $A: \mathcal{H} \rightarrow \mathcal{K}$ will often be denoted as $A_{\mathcal{H} \rightarrow \mathcal{K}}$, and will be identified without further comment with any operator of the form $A \otimes \mathbb{1}_{\mathcal{L}}$ where $\mathbb{1}_{\mathcal{L}}$ is the identity operator on some other Hilbert space $\mathcal{L}$. If $|\psi\rangle \in \mathcal{L}$, the transformation $|\psi\rangle \otimes A: \mathcal{H} \rightarrow \mathcal{K} \otimes \mathcal{L}$ is defined by

$$
\left(|\psi\rangle_{\mathcal{L}} \otimes A_{\mathcal{H} \rightarrow \mathcal{K}}\right)|\phi\rangle_{\mathcal{H}}=|\psi\rangle_{\mathcal{L}} \otimes|A \phi\rangle_{\mathcal{K}}
$$

When $\mathcal{A}: B^{1}(\mathcal{H}) \rightarrow B^{1}(\mathcal{K})$ is a quantum operation, it will often be denoted as $\mathcal{A}_{\mathcal{H} \rightarrow \mathcal{K}}$.

\subsection{Basis-free constructions using dual spaces and double kets}

As in a number of previous works on channel-adapted quantum error recovery [15-17], in Section 5 and in the Appendices A and B it will prove convenient to treat Hilbert spaces and their duals on equal footing:

Definition 25 The dual space $\mathcal{H}^{*}$ of the Hilbert space $\mathcal{H}$ is the set of linear functionals $\bar{\psi}: \mathcal{H} \rightarrow \mathbb{C}$ of the form

$$
\bar{\psi}(\phi):=\langle\psi, \phi\rangle: \mathcal{H} \rightarrow \mathbb{C},
$$

where $\psi \in \mathcal{H}$. The space $\mathcal{H}^{*}$ is a Hilbert space in its own right with inner product

$$
\left\langle\bar{\psi}_{1}, \bar{\psi}_{2}\right\rangle_{\mathcal{H}^{*}}:=\overline{\left(\left\langle\psi_{1}, \psi_{2}\right\rangle_{\mathcal{H}}\right)}=\left\langle\psi_{2}, \psi_{1}\right\rangle_{\mathcal{H}},
$$

where the bar in the middle denotes complex conjugation.

Use of the dual space as a Hilbert space in its own right has pleasant computational properties which are amenable to Dirac notation. ${ }^{[109]}$ For example, if $\psi \in \mathcal{H}$ has the coordinate expansion

$$
\psi=\sum \psi_{i}|i\rangle_{\mathcal{H}}
$$

then the dual vector $\bar{\psi} \in \mathcal{H}^{*}$ has the expansion

$$
\bar{\psi}=\sum \bar{\psi}_{i}|\bar{\imath}\rangle_{\mathcal{H}^{*}},
$$

where the coordinates $\bar{\psi}_{i}:=\overline{\langle i|}_{\mathcal{H}^{*}} \overline{\psi\rangle}_{\mathcal{H}^{*}}$ are simply the complex conjugates of the coordinates $\psi_{i}$ :

$$
\bar{\psi}_{i}=\overline{\left(\psi_{i}\right)} .
$$

Given the linear transformation $A \in B^{2}(\mathcal{H} \rightarrow \mathcal{K})$

$$
A_{\mathcal{H} \rightarrow \mathcal{K}}=\sum A_{i j}|i\rangle_{\mathcal{K}}\left\langle\left. j\right|_{\mathcal{H}}\right.
$$

one may form the conjugate operator $\bar{A}: \mathcal{H}^{*} \rightarrow \mathcal{K}^{*}$, the transpose $A^{\operatorname{tr}}: \mathcal{K}^{*} \rightarrow \mathcal{H}^{*}$, and the basis-free double ket $|A\rangle\rangle_{\mathcal{K H}^{*}} \in \mathcal{K} \otimes \mathcal{H}^{*}$ by

$$
\begin{aligned}
\bar{A}_{\mathcal{H}^{*} \rightarrow \mathcal{K}^{*}} & =\sum \bar{A}_{i j}|\bar{\imath}\rangle_{\mathcal{K}^{*}}\left\langle\left.\bar{j}\right|_{\mathcal{H}^{*}}\right. \\
A_{\mathcal{K}^{*} \rightarrow \mathcal{H}^{*}}^{\mathrm{tr}} & =\sum A_{i j}|\bar{j}\rangle_{\mathcal{H}^{*}}\left\langle\left.\bar{\imath}\right|_{\mathcal{K}^{*}}\right. \\
|A\rangle\rangle_{\mathcal{K H}^{*}} & =\sum A_{i j}|i\rangle_{\mathcal{K}}|\bar{j}\rangle_{\mathcal{H}^{*}} .
\end{aligned}
$$

These equations may be replaced by basis-independent definitions, since they are uniquely-specified by the identities $\bar{A} \bar{\phi}=\overline{(A \phi)}, A^{\text {tr }}=\bar{A}^{\dagger}$, and $\left\langle\psi_{\mathcal{K}} \mid\left\langle\bar{\phi}_{\mathcal{H}^{*}}|| A\right\rangle\right\rangle_{\mathcal{K H}^{*}}=\langle\psi, A \phi\rangle$, for $\phi \in \mathcal{H}$ and $\psi \in \mathcal{K}$, respectively.

The basis-free double bra

$$
\left\langle\left\langle\left. A\right|_{\mathcal{K H}^{*}}=\sum \bar{A}_{i j}\left\langlei | _ { \mathcal { K } } \left\langle\left.\bar{j}\right|_{\mathcal{H}^{*}}\right.\right.\right.\right.
$$


denotes the linear functional on $\mathcal{K} \otimes \mathcal{H}^{*}$ corresponding to $\left.|A\rangle\right\rangle$. The partial transpose is the isometric extension of the mapping $A_{\mathcal{H} \rightarrow \mathcal{K}} \otimes B_{\mathcal{L} \rightarrow \mathcal{M}} \mapsto A_{\mathcal{K}^{*} \rightarrow \mathcal{H}^{*}}^{\text {tr }} \otimes B_{\mathcal{L} \rightarrow \mathcal{M}}$, i.e.

$$
\operatorname{PT}_{B^{2}(\mathcal{H} \rightarrow \mathcal{K}) \rightarrow B^{2}\left(\mathcal{K}^{*} \rightarrow \mathcal{H}^{*}\right)}\left(\sum X_{m k h \ell}\left|m_{\mathcal{M}}\right\rangle\left|k_{\mathcal{K}}\right\rangle\left\langle h_{\mathcal{H}}\left|\left\langle\ell_{\mathcal{L}}\right|\right)=\sum X_{m k h \ell} \mid m_{\mathcal{M}}\right\rangle\left|\bar{h}_{\mathcal{H}^{*}}\right\rangle\left\langle\bar{k}_{\mathcal{K}^{*}}\right|\left\langle\ell_{\mathcal{L}}\right|,\right.
$$

which maps $B^{2}(\mathcal{H} \otimes \mathcal{L} \rightarrow \mathcal{K} \otimes \mathcal{M}) \rightarrow B^{2}\left(\mathcal{K}^{*} \otimes \mathcal{L} \rightarrow \mathcal{H}^{*} \otimes \mathcal{M}\right)$, where $\mathcal{L}$ and $\mathcal{M}$ are arbitrary Hilbert spaces.

We collect some useful identities involving basis-free double-kets:

Lemma 26 1. If $A, B: \mathcal{H} \rightarrow \mathcal{K}$ then

$$
\langle\langle A, B\rangle\rangle_{\mathcal{K H}^{*}}=\operatorname{Tr} A^{\dagger} B
$$

2. Let $A: \mathcal{K} \rightarrow \mathcal{L}, B: \mathcal{H} \rightarrow \mathcal{M}$, and $C: \mathcal{H} \rightarrow \mathcal{K}$. Then

$$
\left.\left.\left(A_{\mathcal{K} \rightarrow \mathcal{L}} \otimes \bar{B}_{\mathcal{H}^{*} \rightarrow \mathcal{M}^{*}}\right)|C\rangle\right\rangle_{\mathcal{K H}^{*}}=\left|A C B^{\dagger}\right\rangle\right\rangle_{\mathcal{L M}^{*}}
$$

3. Let $A: \mathcal{H} \rightarrow \mathcal{L}$ and $B: \mathcal{K} \rightarrow \mathcal{L}$. Then

$$
\left.\left\langle\left\langle\left. A\right|_{\mathcal{L H}^{*}} \times \mid B\right\rangle_{\mathcal{L} \mathcal{K}^{*}}=\operatorname{Tr}_{\mathcal{L}} \mid B\right\rangle\right\rangle_{\mathcal{L} \mathcal{K}^{*}}\left\langle\left\langle\left. A\right|_{\mathcal{L H}^{*}}=\overline{B^{\dagger} A}\right.\right.
$$

4. Let $A: \mathcal{H} \rightarrow \mathcal{K}$ and $B: \mathcal{H} \rightarrow \mathcal{L}$. Then

$$
\begin{gathered}
\left.\operatorname{Tr}_{\mathcal{H}^{*}}|A\rangle\right\rangle_{\mathcal{K H}^{*}}\left\langle\left\langle\left. B\right|_{\mathcal{L H}^{*}}=A_{\mathcal{H} \rightarrow \mathcal{K}} B_{\mathcal{L} \rightarrow \mathcal{H}}^{\dagger}\right.\right. \\
\operatorname{PT}_{B^{2}\left(\mathcal{H}^{*}\right) \rightarrow B^{2}(\mathcal{H})}(|A\rangle\rangle_{\mathcal{K H}^{*}}\left\langle\left\langle\left. B\right|_{\mathcal{L} \mathcal{H}^{*}}\right)=B_{\mathcal{L} \rightarrow \mathcal{H}}^{\dagger} \otimes A_{\mathcal{H} \rightarrow \mathcal{K}}: \mathcal{H} \otimes \mathcal{L} \rightarrow \mathcal{H} \otimes \mathcal{K}\right.
\end{gathered}
$$

Note that by multilinearity it is enough to check these identities for rank-1 operators.

Definition 27 The canonical purification [110] of a quantum state $\rho \in B^{1}(\mathcal{H})$ is given by

$$
\left.\left|\psi_{\rho}\right\rangle=|\sqrt{\rho}\rangle\right\rangle_{\mathcal{H H}^{*}}
$$

When $\mathcal{K}$ is finite-dimensional, ${ }^{[11]}$ the Choi matrix [88] of a transformation $\mathcal{R}: B^{1}(\mathcal{K}) \rightarrow B^{1}(\mathcal{L})$ is given by

$$
\tilde{\mathcal{R}}=\mathcal{R}(|\mathbb{1}\rangle\rangle_{\mathcal{K} \mathcal{K}^{*}}\langle\langle\mathbb{1}|) \in B^{1}\left(\mathcal{L} \mathcal{K}^{*}\right) .
$$

Note that by (64) and (63), $\psi_{\rho}$ has the standard defining property

$$
\rho=\operatorname{Tr}_{\mathcal{H}^{*}}\left|\psi_{\rho}\right\rangle\left\langle\psi_{\rho}\right|
$$

of a purification of $\rho$, and also of $\bar{\rho}$

$$
\bar{\rho}=\operatorname{Tr}_{\mathcal{H}}\left|\psi_{\rho}\right\rangle\left\langle\psi_{\rho}\right| .
$$

In particular, if $\mathcal{H}$ is finite-dimensional then the state $\left.(\operatorname{dim} \mathcal{H})^{-1 / 2}|\mathbb{1}\rangle\right\rangle_{\mathcal{H} \mathcal{H}^{*}}$ is maximally-entangled, and indeed the singular value decomposition of an operator

$$
A=\sum \lambda_{i}\left|f_{i}\right\rangle\left\langle g_{i}\right|
$$

corresponds precisely to the Schmidt decomposition of its double-ket

$$
|A\rangle\rangle=\sum \lambda_{i}\left|f_{i}\right\rangle\left|\bar{g}_{i}\right\rangle .
$$

Remark: A basis-free construction of the Stinespring dilation may be found in Appendix A. 


\section{Minimum-error distinction as a maximal seminorm prob- lem}

The minimum-error quantum detection problem of Definition 1 may be reformulated as a maximalseminorm problem using the identity

$$
P_{\text {succ }}(M)=\|E\|_{\mathcal{E}}^{2},
$$

per the following definition:

Definition 28 Let $\mathcal{E}=\left\{\rho_{k}\right\}_{k \in K}$ be the ensemble of Definition 1. A vector of operators $E=$ $\left\{E_{k}: \mathcal{H} \rightarrow \mathcal{H}\right\}_{k \in K}$ is a generalized measurement (GM) [24] corresponding to the POVM $M=$ $\left\{M_{k}\right\}_{k \in K}$ if one has the decomposition

$$
M_{k}=E_{k}^{\dagger} E_{k} .
$$

The $\mathcal{E}$-semi-inner product is defined for vectors of operators $F=\left\{F_{k}: \mathcal{H} \rightarrow \mathcal{H}\right\}_{k \in K}$ and $G=$ $\left\{G_{k}: \mathcal{H} \rightarrow \mathcal{H}\right\}_{k \in K}$ by

$$
\langle F, G\rangle_{\mathcal{E}}=\operatorname{Tr} \sum_{k \in K} F_{k}^{\dagger} G_{k} \rho_{k} .
$$

The $\mathcal{E}$-semi-inner product space is the space $V_{\mathcal{E}}=\left\{E \mid\|E\|_{\mathcal{E}}<\infty\right\}$, on which $\langle\bullet, \bullet\rangle_{\mathcal{E}}$ is welldefined. The set $S_{\mathcal{E}} \subseteq V_{\mathcal{E}}$ will denote the set of generalized measurements of $\mathcal{E}$.

Remark: It is important to note that if $\mathcal{E}$ is a perfectly distinguishable ensemble of more than one element then $\|\bullet\|_{\mathcal{E}}$ is only a seminorm. In particular, any cyclic permutation $E^{\prime}$ of a perfectlydistinguishing generalized measurement must satisfy $\left\|E^{\prime}\right\|=0$.

\subsection{Computation of directional iterates}

Our first step is to compute directional iterates for generalized measurements:

Theorem 29 (Directional iteration for generalized measurements) Take $S=S_{\mathcal{E}}$ and $V=$ $V_{\mathcal{E}}$ as in Def. 6. Then a directional iterate of $E \in V_{\mathcal{E}}$ is given by

$$
E_{k}^{(+)}=E_{k} \rho_{k}\left(\sum \rho_{\ell} E_{\ell}^{\dagger} E_{\ell} \rho_{\ell}\right)^{-1 / 2^{+}},
$$

where the exponent is given by equation 18. Furthermore, one has the identity

$$
\left\langle E^{(+)}, E\right\rangle_{\mathcal{E}}=\operatorname{Tr} \sqrt{\sum \rho_{k} M_{k} \rho_{k}} .
$$

Remark: It follows by comparison of eq. (73) with eq. (17) that the iteration $E \mapsto E^{(+)}$for GMs corresponds to Ježek, Řeháček, and Fiurášek's iteration $M \mapsto M^{\oplus}$ for POVMs. In particular, $P_{\text {succ }}\left(M^{\oplus}\right) \geq P_{\text {succ }}(M)$, as was observed numerically in [74].

Proof. The proof is an easy modification of that of Theorem 9 of [22], which employs the $E_{k}=\mathbb{1}$ special case of (73). One has the identity

$$
\operatorname{Re}\langle E, F\rangle_{\mathcal{E}}=\operatorname{Re} \operatorname{Tr} V_{E}^{\dagger} U_{F},
$$

where $V_{E}, U_{F}: \mathcal{H} \rightarrow \mathcal{H} \otimes \mathbb{C}^{K}$ are defined by

$$
\begin{aligned}
V_{E} \psi & =\sum_{k \in K}\left(E_{k} \rho_{k} \psi\right) \otimes|k\rangle_{\mathbb{C}^{K}} \\
U_{F} \psi & =\sum_{k \in K}\left(F_{k} \psi\right) \otimes|k\rangle_{\mathbb{C}^{K}},
\end{aligned}
$$


where $|k\rangle_{\mathbb{C}^{K}}$ is the standard basis of $\mathbb{C}^{K}$. Then $F$ is a generalized measurement iff $U_{F}$ is a contraction, with $\left\|U_{F}\right\| \leq 1$. But a contraction $U_{F}$ maximizing (75) is computed using equation 44

$$
U_{F} \psi=V_{E}\left(V_{E}^{\dagger} V_{E}\right)^{-1 / 2^{+}} \psi=\sum|k\rangle_{\mathbb{C}^{K}} \otimes E_{k} \rho_{k}\left(\sum \rho_{\ell} E_{\ell}^{\dagger} E_{\ell} \rho_{\ell}\right)^{-1 / 2^{+}} \psi .
$$

Equations (73) and (74) follow.

\subsection{A "small-angle" guess}

In order to use Lemma 7 to prove distinguishability bounds, one must construct a guess $G$ subtending a provably-small angle with an optimal generalized measurement $E^{\text {opt }}$. As a hint of how to proceed, consider the case that the ensemble $\mathcal{E}=\mathcal{E}^{\mathrm{PD}}$ is a perfectly-distinguishable ensemble, consisting of states $\rho_{k}$ of mutually-orthogonal support. An optimal GM is simply given by

$$
E_{k}^{\mathrm{opt}}=\Pi_{+}\left(\rho_{k}\right),
$$

where the positive projection on the right was defined in (45). Use of spectral theory may be avoided, however, if one notes that the semi-inner product $\langle E, F\rangle_{\mathcal{E}}$ of equation 72 is sensitive to the action of the $E_{k}$ and $F_{k}$ only on the ranges of the corresponding $\rho_{k}$. In particular, the simplest-possible "guess"

$$
G_{k}=\mathbb{1} \text { for all } k
$$

satisfies $G \equiv E^{\mathrm{opt}} \bmod \langle\bullet, \bullet\rangle_{\mathcal{E}}$, since

$$
\left\|E^{\mathrm{opt}}-G\right\|_{\mathcal{E}^{\mathrm{PD}}}^{2}=\operatorname{Tr} \sum\left(\Pi_{+}\left(\rho_{k}\right)-\mathbb{1}\right)^{2} \rho_{k}=0 .
$$

This equation suggests that the guess (77) will remain appropriate for "reasonably distinguishable" ensembles. Indeed, equation 73 shows that the iterate $G^{(+)}$corresponds to the mixed-state generalization of Holevo's asymptotically optimal measurement (24). ${ }^{[12]}$ Furthermore, one obtains the following "small angle" estimates:

Lemma 30 Define $G \in V_{\mathcal{E}}$ by equation 77, and let $M$ be a POVM of non-zero success rate. Then one can decompose $M_{k}=E_{k}^{\dagger} E_{k}$ in such a way that $\langle G, E\rangle_{\mathcal{E}} \in \mathbb{R}$ and

$$
\cos (\theta):=\frac{\langle G, E\rangle_{\mathcal{E}}}{\|G\|_{\mathcal{E}}\|E\|_{\mathcal{E}}} \geq \sqrt{P_{\text {succ }}(M)}
$$

Proof. Chose $M_{k}=\tilde{E}_{k}^{\dagger} \tilde{E}_{k}$ arbitrarily. By the polar decomposition, there exist unitary $U_{k}: \mathcal{H} \rightarrow \mathcal{H}$ so that $U_{k} \tilde{E}_{k} \rho_{k} \geq 0$ for all $k$. Setting

$$
E_{k}=U_{k} \tilde{E}_{k}
$$

it follows from Hölder inequality's (42) that

$$
\langle G, E\rangle_{\mathcal{E}}=\operatorname{Tr} \sum E_{k} \rho_{k}=\sum\left\|E_{k} \rho_{k}\right\|_{1} \geq \sum\left|\operatorname{Tr} E_{k}^{\dagger} E_{k} \rho_{k}\right|=P_{\text {succ }}(M) .
$$

Using the fact that $\|G\|_{\mathcal{E}}=1$, the conclusion follows by dividing both sides by $\|E\|_{\mathcal{E}}=\sqrt{P_{\text {succ }}(M)}$.

Remark: Note that if one rescales $G$ into generalized measurement, as is only possible when the index set is finite, then one obtains the "random guessing" measurement $\tilde{G}_{k}=\mathbb{1} / \sqrt{|K|}$. The reader may therefore be surprised that the first iterate $\tilde{G}^{(+)}$(which is unaffected by rescaling of $G$ ) corresponds to Holevo's asymptotically-optimal measurement, since this guess isn't even "smart" enough to take into account the a-priori probabilities of the $\rho_{k}$ !

The resolution of this paradox is that the unrescaled guess $G_{k}=\mathbb{1}$ does NOT correspond to random guessing: it guesses every value $k$. Equation 78 is therefore analogous to the statement that the teacher who grades a multiple-choice test by means of a punched overlay (with holes only for the correct answers) will give top marks to the daring schoolboy who marks ALL of the ovals on his exam. 


\subsection{A simple proof of the generalized Holevo-Curlander bounds}

We have assembled all the pieces necessary to apply Lemma 7:

Proof of Theorem 15. Take $V=V_{\mathcal{E}}$ and $S=S_{\mathcal{E}}$ to be as in Definition 28, and let $G$ be given by (77). By Theorem 29, one has

$$
\left(G^{(+)}\right)^{\dagger} G^{(+)}=M^{\mathrm{QW}}
$$

By Lemma 30 we may decompose $M_{k}^{\text {opt }}=\left(E_{k}^{\text {opt }}\right)^{\dagger} E_{k}^{\text {opt }}$ in such a way that the "small angle" estimate (79) holds in the equivalent form

$$
\left\langle E^{\mathrm{opt}}, G\right\rangle_{\mathcal{E}} \geq P_{\text {succ }}\left(M^{\mathrm{opt}}\right)
$$

given by (80). Replacing $x_{\max }$ by $E_{k}^{\text {opt }}$ in (13) gives

$$
\sqrt{P_{\text {succ }}\left(M^{\mathrm{opt}}\right)} \geq \sqrt{P_{\text {succ }}\left(M^{\mathrm{QW}}\right)} \geq \Lambda(G) \geq\left\langle E^{\mathrm{opt}}, G\right\rangle_{\mathcal{E}}
$$

where we have used the fact that $\|G\|_{\mathcal{E}}=1$. But by equations (14), (74), and (28),

$$
\Lambda(G)=\operatorname{Tr} \sqrt{\sum \rho_{k}^{2}}=\Lambda
$$

The last inequality of (27) follows by appending (82) to (83). The remaining three inequalities of (27) follow by squaring (83). The inequality $\Lambda \leq 1$ follows by (27).

\section{Maximum overlap as a maximal-seminorm problem}

The maximum overlap problem of equation 1 may be expressed as a maximal seminorm problem using the identity

$$
\operatorname{Tr}_{\mathcal{L} \mathcal{H}}\left(M_{\mathcal{L H}} R_{\mathcal{K} \rightarrow \mathcal{L}}\left(\mu_{\mathcal{K} \mathcal{H}}\right)\right)=\|U\|_{\mu, M}^{2}
$$

where $U$ is a Stinespring dilation of $\mathcal{R}$ and the seminorm is from the following definition:

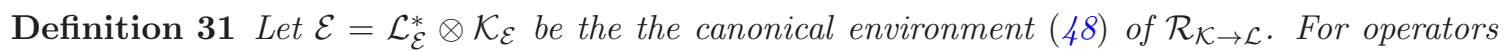
$A, B: \mathcal{H} \rightarrow \mathcal{L} \otimes \mathcal{E}$, the $\mu$-M semi-inner product is defined by

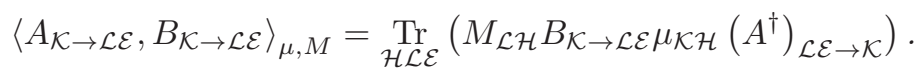

The $\mu$-M semi-inner product space is the space $V_{\mu, M}=\left\{U: \mathcal{K} \rightarrow \mathcal{L} \mathcal{E} \mid\|U\|_{\mu, M}<\infty\right\}$, on which $\langle\bullet, \bullet\rangle_{\mu, M}$ is well-defined. The purification ball is the set $S=\{U: \mathcal{K} \rightarrow \mathcal{L} \mathcal{E} \mid\|U\| \leq 1\} \subseteq$ $V_{\mu, M}$, where $\|\bullet\|$ is the operator-norm.

\subsection{Computation of directional iterates}

As in the case of measurements, it is not difficult to compute directional iterates:

Theorem 32 (Directional iteration for maximum overlap is JFH iteration) Let $V=V_{\mu, M}$ and $S$ be as in Definition 31. Then the operator $U_{\mathcal{K} \rightarrow \mathcal{L E}} \in V_{\mu, M}$ has the directional iterate

$$
U_{\mathcal{K} \rightarrow \mathcal{L E}}^{(+)}=Q\left(Q^{\dagger} Q\right)^{-1 / 2^{+}}
$$

where

$$
Q_{\mathcal{K} \rightarrow \mathcal{L E}}=\operatorname{Tr}_{\mathcal{H}}\left(M_{\mathcal{L H}} U_{\mathcal{K} \rightarrow \mathcal{L E}} \mu_{\mathcal{K} \mathcal{H}}\right)
$$

Furthermore, one has

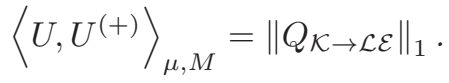




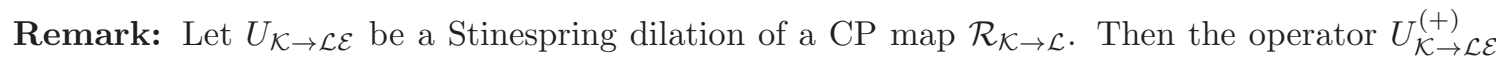
of the above Theorem is a dilation of the Ježek-Fiurášek-Hradil iterate [6, 75], already mentioned in section 1.4.2.

Proof. By cyclicity of the trace and equations (43)-(44),

$$
\begin{aligned}
\max _{W_{\mathcal{K} \rightarrow \mathcal{L} \in S} \in \operatorname{Re}\langle U, W\rangle_{\mu, M}} & =\max _{\|W\| \leq 1} \operatorname{Re} \operatorname{Tr}_{\mathcal{H} \mathcal{L} \mathcal{E}}\left(M_{\mathcal{L H}} W_{\mathcal{K} \rightarrow \mathcal{L E}} \mu_{\mathcal{K} \mathcal{H}}\left(U^{\dagger}\right)_{\mathcal{L E} \rightarrow \mathcal{K}}\right) \\
& =\max _{\|W\| \leq 1} \operatorname{Re} \operatorname{Tr}_{\mathcal{K}}\left(\left(Q^{\dagger}\right)_{\mathcal{L E} \rightarrow \mathcal{K}} W_{\mathcal{K} \rightarrow \mathcal{L E}}\right) \\
& =\| Q_{\mathcal{K} \rightarrow \mathcal{L E} \|_{1}}
\end{aligned}
$$

with maximizer $W=U^{(+)}$given by $(86)$.

\subsection{The restricted maximum-overlap problem}

The remainder of this work restricts consideration to the case that

$$
M_{\mathcal{L H}}=|\phi\rangle_{\mathcal{L H}}\langle\phi|
$$

is a rank 1 projection, seeking to estimate

$$
\operatorname{MO}\left(\mu_{\mathcal{K} \mathcal{H}}, \phi_{\mathcal{L H}}\right):=\sup _{\mathcal{R}}\left\langle\phi_{\mathcal{L H}}\left|\mathcal{R}_{\mathcal{K} \rightarrow \mathcal{L}}\left(\mu_{\mathcal{K} \mathcal{H}}\right)\right| \phi_{\mathcal{L H}}\right\rangle
$$

where the supremum is over quantum operations $\mathcal{R}$ from $\mathcal{K}$ to $\mathcal{L}$. For convenience, we denote

$$
\left\langle A_{\mathcal{K} \rightarrow \mathcal{L E}}, B_{\mathcal{K} \rightarrow \mathcal{L E}}\right\rangle_{\mu, \phi}:=\langle A, B\rangle_{\mu,|\phi\rangle\langle\phi|}=\left\langle\phi_{\mathcal{L H}}\left|\operatorname{Tr}_{\mathcal{E}}\left(B_{\mathcal{K} \rightarrow \mathcal{L E}} \mu_{\mathcal{K} \mathcal{H}}\left(A^{\dagger}\right)_{\mathcal{L E} \rightarrow \mathcal{K}}\right)\right| \phi_{\mathcal{L H}}\right\rangle
$$

and $V_{\mu, \phi}=V_{\mu,|\phi\rangle\langle\phi|}$.

It is worth mentioning that by Theorems 1 and 2 of [70] (see also equation 182 of the appendix), the minimum-error detection problem is a special case of the restricted maximum overlap problem. The importance of this fact for this work is as follows: One may use the study of quantum measurements as a testing ground to for techniques for the study of the maximum overlap problem and its special cases, including quantum error recovery. Furthermore, as we have already seen, Barnum and Knill [14] considered channel reversibility in the sense of average entanglement fidelity by generalizing the "pretty good" measurement.

\subsubsection{A minor simplification}

We use the following notation for the partial traces of $\left|\phi_{\mathcal{L H}}\right\rangle$ :

$$
\begin{aligned}
& \phi_{\mathcal{L}}=\operatorname{Tr}_{\mathcal{H}}|\phi\rangle_{\mathcal{L H}}\langle\phi| \\
& \phi_{\mathcal{H}}=\operatorname{Tr}_{\mathcal{L}}|\phi\rangle_{\mathcal{L H}}\langle\phi|
\end{aligned}
$$

Using the identity

$$
\left|\phi_{\mathcal{L H}}\right\rangle=\Pi_{+}\left(\phi_{\mathcal{H}}\right)\left|\phi_{\mathcal{L H}}\right\rangle
$$

where the positive projection $\Pi_{+}\left(\phi_{\mathcal{H}}\right)$ is given by equation 45 , one has the following

Observation 33 One has the identity

$$
\left\langle\phi_{\mathcal{L H}}\left|\mathcal{R}_{\mathcal{K} \rightarrow \mathcal{L}}\left(\mu_{\mathcal{K} \mathcal{H}}\right)\right| \phi_{\mathcal{L} \mathcal{H}}\right\rangle=\left\langle\phi_{\mathcal{L H}}\left|\mathcal{R}_{\mathcal{K} \rightarrow \mathcal{L}}\left(\hat{\mu}_{\mathcal{K H}}\right)\right| \phi_{\mathcal{L H}}\right\rangle
$$

for any $\mathcal{R}$, where $\hat{\mu}$ is defined by

$$
\hat{\mu}_{\mathcal{K H}}=\Pi_{+}\left(\phi_{\mathcal{H}}\right) \times \mu_{\mathcal{K} \mathcal{H}} \times \Pi_{+}\left(\phi_{\mathcal{H}}\right) .
$$




\subsubsection{A "small angle" guess}

The strategy of Sec. 1.4 calls for construction of a guess $G \in V_{\mu, \phi}$ subtending a provably small angle with some dilation $W^{\text {opt }}: \mathcal{K} \rightarrow \mathcal{L} \otimes \mathcal{E}$ of an optimal overlap operation $\mathcal{R}^{\text {opt }}$. We will be most concerned with the "reasonably overlappable" case, for which one has the crude approximation

$$
\sup _{\mathcal{R}}\left\langle\phi_{\mathcal{L H}}\left|\mathcal{R}_{\mathcal{K} \rightarrow \mathcal{L}}^{\mathrm{opt}}\left(\hat{\mu}_{\mathcal{K H}}\right)\right| \phi_{\mathcal{L H}}\right\rangle \approx \operatorname{Tr} \hat{\mu}_{\mathcal{K H}} .
$$

Our choice of guess will therefore be motivated by the case in which exact equality is obtained:

Proposition 34 (The perfectly overlappable case) Let $\left|\phi_{\mathcal{L H}}\right\rangle$ be a unit vector and let $\mathcal{R}$ be a quantum operation. Then one has perfect overlap

$$
\left\langle\phi_{\mathcal{L H}}\left|\mathcal{R}_{\mathcal{K} \rightarrow \mathcal{L}}\left(\hat{\mu}_{\mathcal{K H}}\right)\right| \phi_{\mathcal{L H}}\right\rangle=\operatorname{Tr} \hat{\mu}
$$

if and only if

$$
\left.\mathcal{R}_{\mathcal{L} \rightarrow \mathcal{K}}^{\dagger}\left(\left|\phi_{\mathcal{L H}}\right\rangle\left\langle\phi_{\mathcal{L H}}\right|\right)\right|_{\operatorname{Ran}\left(\hat{\mu}_{\mathcal{K H}}\right)}=\mathbb{1},
$$

where the adjoint $\mathcal{R}^{\dagger}$ is given by (47).

Proof. Since

$$
\mathcal{R}^{\dagger}\left(\left|\phi_{\mathcal{L H}}\right\rangle\left\langle\phi_{\mathcal{L H}}\right|\right) \leq \mathcal{R}^{\dagger}(\mathbb{1}) \leq \mathbb{1},
$$

the conclusion follows from (47) and (43)-(44).

In section 3.2 we saw for finite ensembles that a properly-rescaled version of the "daring schoolboy's" guess $\left\{G_{k}=\mathbb{1}\right\}$ could be implemented by "random guessing," without use of any measurement apparatus. This suggests consideration of a guess $G_{\mathcal{K} \rightarrow \mathcal{L E}}$ for which the corresponding (possibly trace-increasing) CP map

$$
\mathcal{R}^{G}(\rho)=\operatorname{Tr}_{\mathcal{E}}\left(G \rho G^{\dagger}\right)
$$

is independent of $\rho$.

The following Lemma shows that an analogue of equation 78 is satisfied by a guess of this kind:

Lemma 35 (Construction of a guess) Let $\phi_{\mathcal{L H}}$ be a unit vector and let $G_{\mathcal{K} \rightarrow \mathcal{L E}}$ be a dilation (101) of the (usually trace-increasing) CP map

$$
\mathcal{R}_{\mathcal{K} \rightarrow \mathcal{L}}^{G}(\rho):=\phi_{\mathcal{L}}^{-1^{+}} \times \operatorname{Tr}(\rho),
$$

where $\mathcal{E}$ is the canonical environment (48). Here we use the notation introduced in equations 18 and 93. Then

1. One has the identity

$$
\left(\mathcal{R}^{G}\right)_{\mathcal{L} \rightarrow \mathcal{K}}^{\dagger}\left(\left|\phi_{\mathcal{L H}}\right\rangle\left\langle\phi_{\mathcal{L} \mathcal{H}}\right|\right)=\Pi_{+}\left(\phi_{\mathcal{H}}\right) \otimes \mathbb{1}_{\mathcal{K} \rightarrow \mathcal{K}}
$$

2. If $\mu$ and $\phi$ are "perfectly overlappable" by a quantum operation $\mathcal{R}=\mathcal{R}^{\text {opt }}$, as in equation 99, then $\mathcal{R}^{\text {opt }}$ has a dilation $W_{\mathcal{K} \rightarrow \mathcal{L} \otimes \mathcal{E}}^{\text {opt }}$ such that

$$
\left\|G-W^{o p t}\right\|_{\mu, \phi}=0 .
$$

Remark: Note that the choice of dilation $G$ does not affect the operation

$$
\mathcal{R}^{(+)}(\rho):=\operatorname{Tr}_{\mathcal{E}} G^{(+)} \rho\left(G^{(+)}\right)^{\dagger},
$$


since the replacement $G \rightarrow U_{\mathcal{E} \rightarrow \mathcal{E}} G_{\mathcal{K} \rightarrow \mathcal{L E}}$, where $U_{\mathcal{E} \rightarrow \mathcal{E}}$ is unitary, simply induces the replacement $G^{(+)} \rightarrow U_{\mathcal{E} \rightarrow \mathcal{E}} G^{(+)}$.

Proof. Equation 103 is trivial. To prove the equation 104, note that equations 47 and 103 imply

$$
G_{\mathcal{L E} \rightarrow \mathcal{K}}^{\dagger}\left|\phi_{\mathcal{L H}}\right\rangle\left\langle\phi_{\mathcal{L H}}\right| G_{\mathcal{K} \rightarrow \mathcal{L E}}=\left(\mathcal{R}^{G}\right)_{\mathcal{L} \rightarrow \mathcal{K}}^{\dagger}\left(\left|\phi_{\mathcal{L H}}\right\rangle\left\langle\phi_{\mathcal{L} \mathcal{H}}\right|\right)=\Pi_{+}\left(\phi_{\mathcal{H}}\right) \otimes \mathbb{1}_{\mathcal{K} \rightarrow \mathcal{K}}
$$

In particular, $\left\langle\phi_{\mathcal{L H}}\right| G_{\mathcal{K} \rightarrow \mathcal{L} \otimes \mathcal{E}}$ restricts to an isometry from $\operatorname{ran}\left(\phi_{\mathcal{H}}\right) \otimes \mathcal{K} \supseteq \operatorname{ran}\left(\hat{\mu}_{\mathcal{K} \mathcal{H}}\right)$ into $\mathcal{E}$. Let $W_{\mathcal{K} \rightarrow \mathcal{L E}}^{\prime}$ be a dilation of $\mathcal{R}^{\mathrm{opt}}$. By Proposition 99 it similarly follows that $\left\langle\phi_{\mathcal{L} \mathcal{H}}\right| W_{\mathcal{K} \rightarrow \mathcal{L E}}^{\prime}$ is also an isometry on $\operatorname{ran}\left(\hat{\mu}_{\mathcal{K} \mathcal{H}}\right)$, implying that there exists a unitary $X_{\mathcal{E} \rightarrow \mathcal{E}}$ such that

$$
X_{\mathcal{E} \rightarrow \mathcal{E}}\left\langle\phi_{\mathcal{L H}}\right| W_{\mathcal{K} \rightarrow \mathcal{L} \otimes \mathcal{E}}^{\prime}=\left\langle\phi_{\mathcal{L H}}\right| G_{\mathcal{K} \rightarrow \mathcal{L} \otimes \mathcal{E}}
$$

on $\operatorname{ran}\left(\hat{\mu}_{\mathcal{K} \mathcal{H}}\right)$. Equation 104 follows from (92) by setting $W_{\mathcal{K} \rightarrow \mathcal{L} \otimes \mathcal{E}}^{\text {opt }}=X_{\mathcal{E} \rightarrow \mathcal{E}} W_{\mathcal{K} \rightarrow \mathcal{L} \otimes \mathcal{E}}^{\prime}$

\subsubsection{Angle Estimates}

The following estimate shows that $G_{\mathcal{K} \rightarrow \mathcal{L E}}$ remains a reasonably-good guess when $\phi$ and $\mu$ are only reasonably-overlappable, c.f. inequality 79 :

Lemma 36 (Angle estimates) Take $\phi_{\mathcal{L H}}$ to be a unit vector, take the CP map $\mathcal{R}_{\mathcal{K}}^{G} \rightarrow \mathcal{L}$ and the guess $G_{\mathcal{K} \rightarrow \mathcal{L} \otimes \mathcal{E}}$ to be as in Lemma 35, and let $\mathcal{R}_{\mathcal{K} \rightarrow \mathcal{L}}$ be any quantum operation for which

$$
\left\langle\phi_{\mathcal{L H}}\left|\mathcal{R}_{\mathcal{K} \rightarrow \mathcal{L}}\left(\mu_{\mathcal{K} \mathcal{H}}\right)\right| \phi_{\mathcal{L H}}\right\rangle>0
$$

Then $\mathcal{R}$ has a Stinespring dilation $W_{\mathcal{K} \rightarrow \mathcal{L E}}$ such that $\langle W, G\rangle_{\mu, \phi} \in \mathbb{R}$ and

$$
\cos (\theta):=\frac{\langle W, G\rangle_{\mu, \phi}}{\|W\|_{\mu, \phi}\|G\|_{\mu, \phi}} \geq \sqrt{\frac{\left\langle\phi_{\mathcal{L H}}\left|\mathcal{R}_{\mathcal{K} \rightarrow \mathcal{L}}\left(\hat{\mu}_{\mathcal{K} \mathcal{H}}\right)\right| \phi_{\mathcal{L H}}\right\rangle}{\left\|\mathcal{R}_{\mathcal{L} \rightarrow \mathcal{K}}^{\dagger}\left(\left|\phi_{\mathcal{L H}}\right\rangle\left\langle\phi_{\mathcal{L H}}\right|\right)\right\|_{\infty} \operatorname{Tr}\left(\hat{\mu}_{\mathcal{K} \mathcal{H}}\right)}}
$$

Here $\hat{\mu}_{\mathcal{K H}}$ is given by (97) and the adjoint $\mathcal{R}^{\dagger}$ is from equation $4 \%$. Furthermore, one has the identities

$$
\begin{aligned}
\|G\|_{\mu, \phi}^{2} & =\operatorname{Tr}\left(\hat{\mu}_{\mathcal{K H}}\right) \\
\left\langle G^{(+)}, G\right\rangle_{\mu, \phi} & =\operatorname{Tr}_{\mathcal{K}} \sqrt{\left\langle\left.\phi\right|_{\mathcal{L H}} \hat{\mu}_{\mathcal{K} \mathcal{H}}^{2} \mid \phi\right\rangle_{\mathcal{L H}}}
\end{aligned}
$$

where $G^{(+)}$is the iterate of $G$ given by Theorem 32.

Remark: Note that $\cos (\theta)=1$ if perfect overlap $\left\langle\left.\phi\right|_{\mathcal{L H}} \mathcal{R}_{\mathcal{K} \rightarrow \mathcal{L}}\left(\hat{\mu}_{\mathcal{K H}}\right) \mid \phi\right\rangle_{\mathcal{L H}}=\operatorname{Tr} \hat{\mu}_{\mathcal{K H}}$ is achieved.

Proof. Equation 107 follows from part 1 of Lemma 35:

$$
\|G\|_{\mu, \phi}^{2}=\left\langle\phi_{\mathcal{L H}}\left|\mathcal{R}_{\mathcal{K} \rightarrow \mathcal{L}}^{G}\left(\hat{\mu}_{\mathcal{K} \mathcal{H}}\right)\right| \phi_{\mathcal{L H}}\right\rangle=\operatorname{Tr}\left(\left(\mathcal{R}^{G}\right)_{\mathcal{L} \rightarrow \mathcal{K}}^{\dagger}\left(\left|\phi_{\mathcal{L} \mathcal{H}}\right\rangle\left\langle\phi_{\mathcal{L H}}\right|\right) \hat{\mu}_{\mathcal{K} \mathcal{H}}\right)=\operatorname{Tr} \hat{\mu}_{\mathcal{K} \mathcal{H}}
$$

To prove the angle estimate (106), note that one has the identity

$$
\left\langle W_{\mathcal{K} \rightarrow \mathcal{L E}}, G\right\rangle_{\mu, \phi}=\operatorname{Tr}_{\mathcal{E}} P_{\mathcal{E} \rightarrow \mathcal{E}}
$$

where

$$
P_{\mathcal{E} \rightarrow \mathcal{E}}=\left\langle\phi_{\mathcal{L H}}\left|G_{\mathcal{K} \rightarrow \mathcal{L E}} \mu_{\mathcal{K} \mathcal{H}}\left(W^{\dagger}\right)_{\mathcal{L E} \rightarrow \mathcal{K}}\right| \phi_{\mathcal{L H}}\right\rangle
$$

Starting with any dilation $W_{\mathcal{K} \rightarrow \mathcal{L E}}$ of $\mathcal{R}$, we may assure that $P_{\mathcal{E} \rightarrow \mathcal{E}}$ is positive semidefinite by a replacement

$$
W_{\mathcal{K} \rightarrow \mathcal{L E}} \rightarrow X_{\mathcal{E} \rightarrow \mathcal{E}} W_{\mathcal{K} \rightarrow \mathcal{L E}}
$$


where the unitary operator $X_{\mathcal{E} \rightarrow \mathcal{E}}$ comes from the polar decomposition of $P_{\mathcal{E} \rightarrow \mathcal{E}}$. It follows that the LHS of (106) is real and maximized over the choice of dilation of $\mathcal{R}$. We claim that there exists a an operator $Z: \mathcal{E} \rightarrow \mathcal{E}$ such that

$$
\begin{aligned}
Z_{\mathcal{E} \rightarrow \mathcal{E}} P_{\mathcal{E} \rightarrow \mathcal{E}} & =\left\langle\phi_{\mathcal{L H}}\left|W_{\mathcal{K} \rightarrow \mathcal{L E}} \mu_{\mathcal{K H}}\left(W^{\dagger}\right)_{\mathcal{L E} \rightarrow \mathcal{K}}\right| \phi_{\mathcal{L H}}\right\rangle \\
\left\|Z_{\mathcal{E} \rightarrow \mathcal{E}}\right\|_{\infty} & =\left\|\mathcal{R}_{\mathcal{L}^{\prime} \rightarrow \mathcal{K}}^{\dagger}\left(\left|\phi_{\mathcal{L}^{\prime} \mathcal{H}}\right\rangle\left\langle\phi_{\mathcal{L}^{\prime} \mathcal{H}}\right|\right)\right\|_{\infty}^{1 / 2}
\end{aligned}
$$

Assuming this claim, Hölder's inequality (42) implies that

$$
\begin{aligned}
\left\langle W_{\mathcal{K} \rightarrow \mathcal{L E}}, G\right\rangle_{\mu, \phi} & =\operatorname{Tr}_{\mathcal{E}} P_{\mathcal{E} \rightarrow \mathcal{E}}=\left\|P_{\mathcal{E} \rightarrow \mathcal{E}}\right\|_{1} \\
& \geq \frac{1}{\|Z\|}\left|\operatorname{Tr}_{\mathcal{E}} Z_{\mathcal{E} \rightarrow \mathcal{E}} P_{\mathcal{E} \rightarrow \mathcal{E}}\right| \\
& =\frac{\left\langle\phi_{\mathcal{L H}}\left|\mathcal{R}_{\mathcal{K} \rightarrow \mathcal{L}}\left(\mu_{\mathcal{K} \mathcal{H}}\right)\right| \phi_{\mathcal{L H}}\right\rangle}{\left\|\mathcal{R}_{\mathcal{L} \rightarrow \mathcal{K}}^{\dagger}\left(\left|\phi_{\mathcal{L H}}\right\rangle\left\langle\phi_{\mathcal{L H}}\right|\right)\right\|_{\infty}^{1 / 2}} .
\end{aligned}
$$

The angle estimate (106) follows by dividing both sides by

$$
\|W\|_{\mu, \phi}=\sqrt{\left\langle\phi_{\mathcal{L H}}\left|\mathcal{R}_{\mathcal{K} \rightarrow \mathcal{L}}\left(\mu_{\mathcal{K} \mathcal{H}}\right)\right| \phi_{\mathcal{L H}}\right\rangle}
$$

and by the square root of equation 107 .

To prove the claims $(111)-(112)$, define

$$
Z_{\mathcal{E} \rightarrow \mathcal{E}}=\left\langle\phi_{\mathcal{L}^{\prime} \mathcal{H}}\left|W_{\mathcal{K} \rightarrow \mathcal{L}^{\prime} \mathcal{E}}\left(G^{\dagger}\right)_{\mathcal{L E} \rightarrow \mathcal{K}}\right| \phi_{\mathcal{L H}}\right\rangle
$$

where $\mathcal{L}^{\prime}$ is a copy of $\mathcal{L}$. Then

$$
\begin{aligned}
\left\|Z_{\mathcal{E} \rightarrow \mathcal{E}}\right\|_{\infty} & =\left\|\left(Z^{\dagger} Z\right)_{\mathcal{E} \rightarrow \mathcal{E}}\right\|_{\infty}^{1 / 2}=\left\|\left\langle\phi_{\mathcal{L H}}\left|G_{\mathcal{K} \rightarrow \mathcal{L E}} W_{\mathcal{L}^{\prime} \mathcal{E} \rightarrow \mathcal{K}}^{\dagger}\right| \phi_{\mathcal{L}^{\prime} \mathcal{H}}\right\rangle\left\langle\phi_{\mathcal{L}^{\prime} \mathcal{H}}\left|W_{\mathcal{K} \rightarrow \mathcal{L}^{\prime} \mathcal{E}}\left(G^{\dagger}\right)_{\mathcal{L E} \rightarrow \mathcal{K}}\right| \phi_{\mathcal{L H}}\right\rangle\right\|_{\infty}^{1 / 2} \\
& =\left\|\left\langle\phi_{\mathcal{L} \mathcal{H}}\left|G_{\mathcal{K} \rightarrow \mathcal{L} \mathcal{E}} \mathcal{R}_{\mathcal{L}^{\prime} \rightarrow \mathcal{K}}^{\dagger}\left(\left|\phi_{\mathcal{L}^{\prime} \mathcal{H}}\right\rangle\left\langle\phi_{\mathcal{L}^{\prime} \mathcal{H}}\right|\right)\left(G^{\dagger}\right)_{\mathcal{L E} \rightarrow \mathcal{K}}\right| \phi_{\mathcal{L H}}\right\rangle\right\|_{\infty}^{1 / 2} .
\end{aligned}
$$

But as in the proof of Lemma 35 , the operator $\left\langle\phi_{\mathcal{L H}}\right| G_{\mathcal{K} \rightarrow \mathcal{L E}}$ is an isometry from $\mathcal{K} \otimes \operatorname{ran}\left(\phi_{\mathcal{H}}\right)$ into $\mathcal{E}$, proving (112).

To prove (111), note that equations $114,105, \& 95$ imply that

$$
Z_{\mathcal{E} \rightarrow \mathcal{E}}\left\langle\phi_{\mathcal{L H}}\right| G_{\mathcal{K} \rightarrow \mathcal{L E}}=\left\langle\phi_{\mathcal{L}^{\prime} \mathcal{H}}\right| W_{\mathcal{K} \rightarrow \mathcal{L}^{\prime} \mathcal{E}} \Pi_{+}\left(\phi_{\mathcal{H}}\right)=\left\langle\phi_{\mathcal{L}^{\prime} \mathcal{H}}\right| W_{\mathcal{K} \rightarrow \mathcal{L}^{\prime} \mathcal{E}}
$$

Equation 111 follows, since by (110)

$$
\begin{aligned}
Z_{\mathcal{E} \rightarrow \mathcal{E}} P_{\mathcal{E} \rightarrow \mathcal{E}} & =Z_{\mathcal{E} \rightarrow \mathcal{E}}\left\langle\phi_{\mathcal{L}^{\prime} \mathcal{H}}\left|G_{\mathcal{K} \rightarrow \mathcal{L}^{\prime} \mathcal{E}} \mu_{\mathcal{K H}}\left(W^{\dagger}\right)_{\mathcal{L E} \rightarrow \mathcal{K}}\right| \phi_{\mathcal{L H}}\right\rangle \\
& =\left\langle\phi_{\mathcal{L H}}\left|W_{\mathcal{K} \rightarrow \mathcal{L E}} \mu_{\mathcal{K} \mathcal{H}}\left(W^{\dagger}\right)_{\mathcal{L E} \rightarrow \mathcal{K}}\right| \phi_{\mathcal{L H}}\right\rangle .
\end{aligned}
$$

It remains to prove equation 108. By equation 88 ,

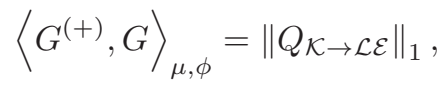

where by equations 87 and 90 ,

$$
\begin{aligned}
Q_{\mathcal{K} \rightarrow \mathcal{L E}} & =\operatorname{Tr}_{\mathcal{H}}\left(\left|\phi_{\mathcal{L H}}\right\rangle\left\langle\phi_{\mathcal{L}^{\prime} \mathcal{H}}\right| G_{\mathcal{K} \rightarrow \mathcal{L}^{\prime} \mathcal{E}} \mu_{\mathcal{K} \mathcal{H}}\right) \\
& =\left\langle\phi_{\mathcal{L}^{\prime} \mathcal{H}}\left|G_{\mathcal{K} \rightarrow \mathcal{L}^{\prime} \mathcal{E}} \mu_{\mathcal{K} \mathcal{H}}\right| \phi_{\mathcal{L H}}\right\rangle
\end{aligned}
$$

where $\mathcal{L}^{\prime}$ is a copy of $\mathcal{L}$. It follows by equations 105 and 95 that

$$
\begin{aligned}
Q^{\dagger} Q & =\left\langle\phi_{\mathcal{L H}}\left|\mu_{\mathcal{K H}} \chi_{+}\left(\phi_{\mathcal{H}}\right) \mu_{\mathcal{K H}}\right| \phi_{\mathcal{L H}}\right\rangle \\
& =\left\langle\phi_{\mathcal{L H}}\left|\hat{\mu}_{\mathcal{K} \mathcal{H}}^{2}\right| \phi_{\mathcal{L H}}\right\rangle .
\end{aligned}
$$

The conclusion follows. 


\subsubsection{The "Quadratic Overlapper"}

The following operation is analogous to the quadratically-weighted measurement:

Definition 37 The "quadratic overlapper" is the operation $\mathcal{R}^{Q O}: B^{1}(\mathcal{K}) \rightarrow B^{1}(\mathcal{L})$ defined by

$$
\mathcal{R}^{Q O}\left(v_{\mathcal{K}}\right)=\operatorname{Tr}_{\mathcal{E}} G_{\mathcal{K} \rightarrow \mathcal{L E}}^{(+)} v_{\mathcal{K}}\left(G_{\mathcal{K} \rightarrow \mathcal{L E}}^{(+)}\right)^{\dagger}
$$

where $G_{\mathcal{K} \rightarrow \mathcal{L E}} \in V_{\mu, \phi}$ is the "small-angle guess" of Lemma 35, with directional iterate $G_{\mathcal{K} \rightarrow \mathcal{L E}}^{(+)}$given by equations 86-87 of Theorem 32.

Alternatively, one may express $\mathcal{R}^{\mathrm{QO}}$ using

Theorem 38 (Computation of the quadratic overlapper) One has the formula

$$
\mathcal{R}_{\mathcal{K} \rightarrow \mathcal{L}}^{Q O}\left(v_{\mathcal{K}}\right)=\operatorname{Tr}_{\mathcal{K} \mathcal{H}}\left(\hat{\mu}_{\mathcal{K} \mathcal{H}}^{2}\left(\left(Y^{-1 / 2^{+}} v Y^{-1 / 2^{+}}\right)_{\mathcal{K} \rightarrow \mathcal{K}} \otimes|\phi\rangle_{\mathcal{L H}}\langle\phi|\right)\right)
$$

where

$$
Y_{\mathcal{K} \rightarrow \mathcal{K}}=\left\langle\phi_{\mathcal{L H}}\left|\hat{\mu}_{\mathcal{K} \mathcal{H}}^{2}\right| \phi_{\mathcal{L H}}\right\rangle
$$

and $\hat{\mu}_{\mathcal{K H}}$ is given by $(97)$.

Proof. By equation 86, the guess $G$ has the iterate

$$
G_{\mathcal{K} \rightarrow \mathcal{L E}}^{(+)}=Q\left(Q^{\dagger} Q\right)^{-1 / 2^{+}}
$$

where the operator $Q$ is defined by (87). By equations (117)-(118), one has

$$
\begin{aligned}
Q_{\mathcal{K} \rightarrow \mathcal{L E}} & =\left\langle\phi_{\mathcal{L}^{\prime} \mathcal{H}}\left|G_{\mathcal{K} \rightarrow \mathcal{L}^{\prime} \mathcal{E}} \mu_{\mathcal{K H}}\right| \phi_{\mathcal{L H}}\right\rangle \\
Q^{\dagger} Q & =\left\langle\phi_{\mathcal{L H}}\left|\hat{\mu}_{\mathcal{K} \mathcal{H} \rightarrow \mathcal{K H}}^{2}\right| \phi_{\mathcal{L H}}\right\rangle=Y_{\mathcal{K} \rightarrow \mathcal{K}} .
\end{aligned}
$$

Setting

$$
\tilde{v}_{\mathcal{K} \rightarrow \mathcal{K}}=Y^{-1 / 2^{+}} v Y^{-1 / 2^{+}}
$$

it follows that

$$
\begin{aligned}
& \operatorname{Tr}_{\mathcal{E}} G_{\mathcal{K} \rightarrow \mathcal{L E}}^{(+)} v_{\mathcal{K}}\left(G^{(+)}\right)_{\mathcal{L E} \rightarrow \mathcal{K}}^{\dagger} \\
& =\operatorname{Tr}_{\mathcal{E}}\left(\left\langle\phi_{\mathcal{L}^{\prime} \mathcal{H}}\left|G_{\mathcal{K} \rightarrow \mathcal{L}^{\prime} \mathcal{E}} \mu_{\mathcal{K H}}\right| \phi_{\mathcal{L H}}\right\rangle \tilde{v}_{\mathcal{K} \rightarrow \mathcal{K}}\left\langle\phi_{\mathcal{L H}}\left|\mu_{\mathcal{K H}}\left(G^{\dagger}\right)_{\mathcal{L}^{\prime} \mathcal{E} \rightarrow \mathcal{K}}\right| \phi_{\mathcal{L}^{\prime} \mathcal{H}}\right\rangle\right) \\
& =\operatorname{Tr}_{\mathcal{K H}}\left(\mu_{\mathcal{K H}}\left|\phi_{\mathcal{L H}}\right\rangle \tilde{v}_{\mathcal{K} \rightarrow \mathcal{K}}\left\langle\phi_{\mathcal{L H}}\left|\mu_{\mathcal{K} \mathcal{H}}\left(G^{\dagger}\right)_{\mathcal{L}^{\prime} \mathcal{E} \rightarrow \mathcal{K}}\right| \phi_{\mathcal{L}^{\prime} \mathcal{H}}\right\rangle\left\langle\phi_{\mathcal{L}^{\prime} \mathcal{H}}\right| G_{\mathcal{K} \rightarrow \mathcal{L}^{\prime} \mathcal{E}}\right) \\
& =\operatorname{Tr}_{\mathcal{K H}}\left(\mu_{\mathcal{K H}}\left(\tilde{v}_{\mathcal{K} \rightarrow \mathcal{K}} \otimes|\phi\rangle_{\mathcal{L H}}\langle\phi|\right) \mu_{\mathcal{K} \mathcal{H}} \Pi_{+}\left(\phi_{\mathcal{H}}\right)\right) \\
& =\operatorname{Tr}_{\mathcal{K H}}\left(\hat{\mu}_{\mathcal{K} \mathcal{H}}^{2}\left(\tilde{v}_{\mathcal{K} \rightarrow \mathcal{K}} \otimes|\phi\rangle_{\mathcal{L H}}\langle\phi|\right)\right) .
\end{aligned}
$$

Here equation 123 uses cyclicity of the trace, equation 124 uses (105), and equation 125 uses (95), (97), and cyclicity of the trace.

\subsubsection{Estimates for the restricted maximum overlap problem}

On now may apply our angle estimates in a manner similar to that of section 3.3:

Theorem 39 (Two-sided estimates for the maximum overlap problem) Let $\mu_{\mathcal{K} \mathcal{H}}$ be positive semidefinite on $\mathcal{K} \otimes \mathcal{H}$ and let $\left|\phi_{\mathcal{L H}}\right\rangle$ be a unit vector. Then

$$
\begin{aligned}
\frac{\Lambda^{2}}{\operatorname{Tr} \hat{\mu}_{\mathcal{K H}}} & \leq\left\langle\phi_{\mathcal{L H}}\left|\mathcal{R}_{\mathcal{K} \rightarrow \mathcal{L}}^{Q O}\left(\mu_{\mathcal{K} \mathcal{H}}\right)\right| \phi_{\mathcal{L H}}\right\rangle \leq \max _{\mathcal{R}_{\mathcal{K} \rightarrow \mathcal{L}}}\left\langle\phi_{\mathcal{L H}}\left|\mathcal{R}_{\mathcal{K} \rightarrow \mathcal{L}}\left(\mu_{\mathcal{K H}}\right)\right| \phi_{\mathcal{L} \mathcal{H}}\right\rangle \\
& \leq \Lambda \times\left\|\left(\mathcal{R}^{o p t}\right)_{\mathcal{L} \rightarrow \mathcal{K}}^{\dagger}\left(\left|\phi_{\mathcal{L H}}\right\rangle\left\langle\phi_{\mathcal{L H}}\right|\right)\right\|_{\infty}^{1 / 2} \leq \Lambda,
\end{aligned}
$$


where the maximum is over quantum operations $\mathcal{R}: B^{1}(\mathcal{K}) \rightarrow B^{1}(\mathcal{L})$, where $\mathcal{R}^{\text {opt }}$ attains this maximum, and where

$$
\Lambda=\operatorname{Tr}_{\mathcal{K}} \sqrt{\left\langle\phi_{\mathcal{L H}}\left|\hat{\mu}_{\mathcal{K} \mathcal{H}}^{2}\right| \phi_{\mathcal{L H}}\right\rangle}
$$

Here $\hat{\mu}_{\mathcal{K H}}$ is given by $(97), \mathcal{R}^{\dagger}$ is given by $\left(4^{7} 7\right)$, and one interprets $0^{2} / 0=0$.

Remark: It follows from (126) that

$$
\Lambda \leq \operatorname{Tr}\left(\hat{\mu}_{\mathcal{K} \mathcal{H}}\right) \times\left\|\left(\mathcal{R}^{\mathrm{opt}}\right)_{\mathcal{L} \rightarrow \mathcal{K}}^{\dagger}\left(\left|\phi_{\mathcal{L H}}\right\rangle\left\langle\phi_{\mathcal{L H}}\right|\right)\right\|_{\infty}^{1 / 2} \leq \operatorname{Tr} \hat{\mu}_{\mathcal{K H}}
$$

Note: Given an arbitrary invertible operator $X: \mathcal{H} \rightarrow \mathcal{H}$, one may obtain potentially sharper estimates from inequality (126) using a replacement of the form

$$
\begin{aligned}
\phi_{\mathcal{L H}} & \rightarrow \frac{X_{\mathcal{H} \rightarrow \mathcal{H}} \phi_{\mathcal{L H}}}{\left\|X_{\mathcal{H} \rightarrow \mathcal{H}} \phi_{\mathcal{L H}}\right\|} \\
\mu_{\mathcal{K H}} & \rightarrow\left\|X_{\mathcal{H} \rightarrow \mathcal{H}} \phi_{\mathcal{L H}}\right\|^{2}\left(X^{-1}\right)^{\dagger} \mu_{\mathcal{K} \mathcal{H}} X^{-1},
\end{aligned}
$$

which does not change the overlap $\left\langle\phi_{\mathcal{L H}}\left|\mathcal{R}_{\mathcal{K} \rightarrow \mathcal{L}}\left(\mu_{\mathcal{K} \mathcal{H}}\right)\right| \phi_{\mathcal{L H}}\right\rangle$.

Proof. Since all quantities in (126) scale linearly in $\mu$, set $\operatorname{Tr} \hat{\mu}_{\mathcal{K H}}=1$. (The case $\hat{\mu}=0$ is trivial.)

Let $\mathcal{R}^{\text {opt }}$ attain the maximum in (126). Take $G_{\mathcal{K} \rightarrow \mathcal{L E}}$ to be the "small angle" guess of Lemma 35, with iterate $G_{\mathcal{K} \rightarrow \mathcal{L E}}^{(+)}$given by Theorem 32. By Lemma 36, there exists a Stinespring dilation $W_{\mathcal{K} \rightarrow \mathcal{L E}}^{\text {opt }}$ of $\mathcal{R}^{\text {opt }}$ such that the angle estimate (106) holds in the equivalent form

$$
\left\langle W^{\mathrm{opt}}, G\right\rangle_{\mu, \phi} \geq \frac{\left\langle\phi_{\mathcal{L H}}\left|\mathcal{R}_{\mathcal{K} \rightarrow \mathcal{L}}^{\mathrm{opt}}\left(\mu_{\mathcal{K} \mathcal{H}}\right)\right| \phi_{\mathcal{L H}}\right\rangle}{\left\|\left(\mathcal{R}^{\mathrm{opt}}\right)_{\mathcal{L} \rightarrow \mathcal{K}}^{\dagger}\left(\left|\phi_{\mathcal{L H}}\right\rangle\left\langle\phi_{\mathcal{L H}}\right|\right)\right\|_{\infty}^{1 / 2}}
$$

given by (113). But by lemma 7 and (107),

$$
\left\|W^{\mathrm{opt}}\right\|_{\mu, \phi} \geq\left\|G^{(+)}\right\|_{\mu, \phi} \geq \Lambda(G) \geq\left\langle W^{\mathrm{opt}}, G\right\rangle_{\mu, \phi}
$$

where by (14) and (107) - (108),

$$
\Lambda(G)=\operatorname{Re}\left\langle G^{(+)}, G /\|G\|_{\mu, \phi}\right\rangle_{\mu, \phi}=\Lambda .
$$

The third inequality of (126) follows by appending (130) to (131). Squaring the first three quantities of (131) proves the first two inequalities of (126). The final inequality follows from the fact that $\mathcal{R}^{\text {opt }}$ is a quantum operation.

\subsection{Estimates for quantum conditional min-entropy}

Theorem 39 has the following corollary:

Corollary 40 Let $\mathcal{H}_{A}$ and $\mathcal{H}_{B}$ be finite-dimensional, and let $\rho_{A B}$ be a density matrix on $\mathcal{H}_{A} \otimes \mathcal{H}_{B}$. Then for any $s \in \mathbb{R}$ the conditional min-entropy (10) of $A$ given $B$ satisfies the bounds

$$
-\log _{2}\left(\sqrt{\operatorname{Tr} \rho_{A}^{s}} \times \operatorname{Tr}_{B} \sqrt{\operatorname{Tr}_{A} \rho_{A B} \rho_{A}^{-s} \rho_{A B}}\right) \leq H_{\min }(A \mid B)_{\rho} \leq-\log _{2}\left(\frac{\left(\operatorname{Tr}_{B} \sqrt{\operatorname{Tr}_{A} \rho_{A B} \rho_{A}^{-s} \rho_{A B}}\right)^{2}}{\operatorname{Tr} \rho_{A}^{1-s}}\right)
$$

where $\rho_{A}=\operatorname{Tr}_{B} \rho_{A B}$. Here any non-positive powers are evaluated as in equation 18. (In particular

$$
\rho_{A}^{0}:=\Pi_{+}\left(\rho_{A}\right)
$$

where the RHS is the positive projection (45).) 


\section{Remarks:}

1. The $s=0$ case of (133) is particularly simple:

$$
-\log \left(\operatorname{Tr}_{B} \sqrt{\operatorname{Tr}_{A} \rho_{A B}^{2}}\right)-\frac{1}{2} \log \left(\operatorname{rank}\left(\rho_{A}\right)\right) \leq H_{\min }(A \mid B)_{\rho} \leq-2 \log _{2}\left(\underset{B}{\operatorname{Tr}} \sqrt{\operatorname{Tr}_{A} \rho_{A B}^{2}}\right) .
$$

2. If $s=1 / 2$ and $\rho_{A B}=\left|\psi_{A B}\right\rangle\left\langle\psi_{A B}\right|$ is pure then a simple calculation ${ }^{[113]}$ shows that the upper and lower bounds of (133) agree, yielding the known [70] expression

$$
H_{\min }(A \mid B)_{\left|\psi_{A B}\right\rangle}=-2 \log _{2}\left(\underset{A}{\operatorname{Tr}} \sqrt{\rho_{A}}\right) .
$$

3. More generally, if $\rho_{A B}=\left|\psi_{A B}\right\rangle\left\langle\psi_{A B}\right|$ is pure then the upper bound of (133) is exact and independent of $s$.

4. If $\rho_{A B}$ is a maximally-entangled pure state then the lower bound of (133) is also exact and independent of $s$.

Proof. Let

$$
\rho_{A}=\sum \lambda_{k}|k\rangle_{A}\langle k|
$$

be a spectral decomposition. Then by equation 12 one has the identity

$$
2^{-H_{\min }(A \mid B)_{\rho}}=\left\langle\left.\psi_{s}\right|_{A A^{*}} \max _{\mathcal{R}}\left(\mathcal{R}_{B \rightarrow A^{*}}\left(\mu_{s}\right)_{A B}\right) \mid \psi_{s}\right\rangle_{A A^{*}},
$$

where

$$
\begin{aligned}
\left|\psi_{s}\right\rangle_{A A^{*}} & =\frac{\left.\left|\rho_{A}^{s / 2}\right\rangle\right\rangle_{A A^{*}}}{\left\|\rho_{A}^{s / 2}\right\|_{2}}=\sum_{k \text { with } \lambda_{k}>0} \frac{\lambda_{k}^{s / 2}}{\sqrt{\operatorname{Tr} \rho_{A}^{s}}}|k\rangle_{A}|\bar{k}\rangle_{A^{*}} \\
\left(\mu_{s}\right)_{A B} & =\left\|\rho_{A}^{s / 2}\right\|_{2}^{2} \rho_{A}^{-s / 2} \rho_{A B} \rho_{A}^{-s / 2} .
\end{aligned}
$$

The bounds (133) follow by Theorem 39 .

Remark: Using the fourth term of inequality 126, one may tighten the lower bound of (133) in cases where one can estimate

$$
\left\|\left(\mathcal{R}^{\mathrm{opt}}\right)_{B \rightarrow \mathcal{A}^{*}}^{\dagger}\left(\left|\psi_{s}\right\rangle\left\langle\psi_{s}\right|\right)\right\|_{\infty},
$$

where $\mathcal{R}^{\text {opt }}$ is a maximizer of (137). Appendix C shows that this works in the case that $\rho_{A B}$ is a "quantum-classical" state.

\section{Approximate Channel Reversals}

This section applies Theorem 39 to estimate the reversibility of an arbitrary quantum operation $\mathcal{A}: B^{1}(\mathcal{H}) \rightarrow B^{1}(\mathcal{K})$, as measured by entanglement fidelity $\max _{\mathcal{R}_{\mathcal{K} \rightarrow \mathcal{H}}} F_{e}(\rho, \mathcal{R} \circ \mathcal{A})$. (Note that more generally Theorem 39 gives estimates when the input state of $\mathcal{A}$ and target output state of $\mathcal{R}$ differ, but we focus on this special case.)

In order to express the our reversibility estimates in a more intuitive form (and to understand the relationship of the corresponding reversal with that of Barnum and Knill), it is useful to introduce a method for applying functions to CP maps. 


\subsection{The $\rho$-functional calculus for CP maps}

One may tailor the Kraus decomposition [89] a CP map to a given input density matrix $\rho$ :

Definition 41 Let $\mathcal{A}: B^{1}(\mathcal{H}) \rightarrow B^{1}(\mathcal{K})$ be a completely-positive map and let $\rho$ be a density matrix on $\mathcal{H}$. A $\rho$-Kraus decomposition of the restriction of $\mathcal{A}$ to $B^{1}(\operatorname{supp}(\rho))$ is a decomposition of the form

$$
\mathcal{A}(\mu)=\sum p_{k} E_{k} \mu E_{k}^{\dagger}, \quad \operatorname{supp}(\mu) \subseteq \operatorname{supp}(\rho)
$$

where

1. The vectors $E_{k}\left|\psi_{\rho}\right\rangle \in \mathcal{K} \otimes \mathcal{H}^{*}$ are orthonormal, where $\psi_{\rho}$ is the purification (66).

2. The $p_{k}$ are non-negative.

3. The $\rho$-Kraus operators $E_{k}: \mathcal{H} \rightarrow \mathcal{K}$ have supports in $\operatorname{supp}(\rho)$.

\section{Remarks:}

1. Existence of a $\rho$-Kraus decomposition follows by an easy modification of standard techniques. (See Proposition 43, below.)

2. When $\mathcal{A}$ is trace-preserving, one interprets $\mathcal{A}$ as acting on $\rho$ by randomly sending the purification $\left|\psi_{\rho}\right\rangle_{\mathcal{H H}^{*}}$ into one of the orthonormal vectors $E_{k}\left|\psi_{\rho}\right\rangle$, which are classically-distinguishable by the observer with access to $\mathcal{K H}^{*}$.

3. By equations 66 and 61 , the orthonormality of the $E_{k}\left|\psi_{\rho}\right\rangle_{\mathcal{H}_{\mathcal{H}^{*}}}$ is equivalent to the condition

$$
\operatorname{Tr}\left(E_{k}^{\dagger} E_{\ell} \rho\right)=\delta_{k \ell}
$$

If $\rho$ is maximally-mixed one therefore obtains the usual orthogonality conditions [90] sometimes required for the Kraus operators.

Given a state $\rho$, there is a natural notion of applying functions to completely positive maps:

Definition 42 ( $\rho$-functional calculus for $\mathbf{C P}$ maps) Let $\mathcal{A}: B^{1}(\mathcal{H}) \rightarrow B^{1}(\mathcal{K})$ be a completely positive map with the $\rho$-Kraus decomposition $(140)$. For $f:[0, \infty) \rightarrow[0, \infty)$ one defines the $C P$ map $f_{\rho}(\mathcal{A}): B^{1}(\operatorname{supp}(\rho)) \rightarrow B^{1}(\mathcal{K})$ by

$$
\left(f_{\rho}(\mathcal{A})\right)(\mu)=\sum f\left(p_{k}\right) E_{k} \mu E_{k}^{\dagger}
$$

The quadratic reweighting $\mathcal{A}^{(2, \rho)}$ of $\mathcal{A}$ corresponds to the case $f(p)=p^{2}$ :

$$
\mathcal{A}^{(2, \rho)}(\mu)=\sum p_{k}^{2} E_{k} \mu E_{k}^{\dagger}
$$

The following proposition shows that the CP maps $f_{\rho}(\mathcal{A})$ and $\mathcal{A}^{(2, \rho)}$ are well-defined and independent of the decomposition (140):

Proposition 43 Let $f, \mathcal{A}$, and $\rho$ be as in Definition 42. Then

1. A $\rho$-Kraus decomposition (140) of $\mathcal{A}$ exists. Furthermore,

$$
\sum p_{k}=\operatorname{Tr} \mathcal{A}(\rho)
$$

so that $\left\{p_{k}\right\}$ is a probability distribution if $\mathcal{A}$ is trace preserving. 
2. One has the identity

$$
\left(f_{\rho}(\mathcal{A})\right)(\mu)=\operatorname{Tr}_{\mathcal{H}^{*}}\left(\left(\overline{\rho^{-1 / 2^{+}} \mu^{\dagger} \rho^{-1 / 2+}}\right)_{\mathcal{H}^{*} \rightarrow \mathcal{H}^{*}} \times f\left(\mathcal{A}\left(\left|\psi_{\rho}\right\rangle_{\mathcal{H} \mathcal{H}^{*}}\left\langle\psi_{\rho}\right|\right)\right)\right),
$$

for all $\mu \in B^{1}(\operatorname{supp}(\rho))$, where barred product of operators acts on $\mathcal{H}^{*}$ as indicated by equations 56-60. Here one applies $f$ to a self-adjoint operator using the functional calculus [86]: Given a spectral decomposition

$$
A=\sum \lambda_{i} \Pi_{i}
$$

one writes

$$
f(A)=\sum f\left(\lambda_{i}\right) \Pi_{i} .
$$

3. In particular, the CP map $f_{\rho}(\mathcal{A})$ is independent of the choice of decomposition (140), and

$$
\mathcal{A}^{(2, \rho)}(\mu)=\operatorname{Tr}_{\mathcal{H}^{*}}\left(\left(\overline{\rho^{-1 / 2^{+}} \mu^{\dagger} \rho^{-1 / 2^{+}}}\right)_{\mathcal{H}^{*} \rightarrow \mathcal{H}^{*}} \times\left(\mathcal{A}\left(\left|\psi_{\rho}\right\rangle_{\mathcal{H} \mathcal{H}^{*}}\left\langle\psi_{\rho}\right|\right)\right)^{2}\right),
$$

for $\mu \in B^{1}(\operatorname{supp}(\rho))$.

\section{Remarks:}

1. If $f(p)=p$ is the identity function and if $\rho$ is maximally mixed then equation 144 reduces to the usual procedure for recovering a channel from its Choi matrix (67).

2. Equation (147) gives the form of $\mathcal{A}^{(2, \rho)}$ which appears when one applies Theorem 39 to obtain reversibility estimates. The transpose of $\mu$ becomes a partial transpose $(60)$ when $\mathcal{A}^{(2, \rho)}$ is applied to the state of a composite quantum system.

3. A prescription for computing $\mathcal{A}^{(2, \rho)}$ from an arbitrary set of Kraus operators of $\mathcal{A}$ appears in the next section.

Proof. By equations 62, 66, 18, and 45,

$$
\left.\left.\overline{\mu^{1 / 2} \rho^{-1 / 2^{+}}}\left|\psi_{\rho}\right\rangle_{\mathcal{H} \mathcal{H}^{*}}=\left|\rho^{1 / 2} \rho^{-1 / 2^{+}} \mu^{1 / 2}\right\rangle\right\rangle_{\mathcal{H H}^{*}}=\left|\Pi_{+}(\rho) \mu^{1 / 2}\right\rangle\right\rangle_{\mathcal{H H}^{*}}=\left|\psi_{\mu}\right\rangle_{\mathcal{H H}^{*}} .
$$

It therefore follows by equation 68 that for densities $\mu \in B^{1}(\mathcal{H})$

$$
\mathcal{A}(\mu)=\operatorname{Tr}_{\mathcal{H}^{*}} \mathcal{A}\left(\left|\psi_{\mu}\right\rangle_{\mathcal{H H}^{*}}\left\langle\psi_{\mu}\right|\right)=\operatorname{Tr}_{\mathcal{H}^{*}} \overline{\mu^{1 / 2} \rho^{-1 / 2+}} \mathcal{A}\left(\left|\psi_{\rho}\right\rangle_{\mathcal{H H}^{*}}\left\langle\psi_{\rho}\right|\right) \overline{\rho^{-1 / 2+} \mu^{1 / 2}}
$$

Taking a spectral decomposition

$$
\left.\mathcal{A}\left(\left|\psi_{\rho}\right\rangle\left\langle\psi_{\rho}\right|\right)=\sum p_{k}\left|F_{k}\right\rangle\right\rangle_{\mathcal{K H}^{*}}\left\langle\left\langle F_{k}\right|,\right.
$$

it follows from equations 149,62 , and 64 that

$$
\left.\mathcal{A}(\mu)=\operatorname{Tr}_{\mathcal{H}^{*}} \sum p_{k}\left|F_{k} \rho^{-1 / 2^{+}} \mu^{1 / 2}\right\rangle\right\rangle_{\mathcal{K H}^{*}}\left\langle\left\langle F_{k} \rho^{-1 / 2^{+}} \mu^{1 / 2}\right|=\sum p_{k} F_{k} \rho^{-1 / 2^{+}} \mu \rho^{-1 / 2^{+}} F_{k}^{\dagger} .\right.
$$

Setting

$$
E_{k}=F_{k} \rho_{k}^{-1 / 2^{+}}
$$

gives the desired $\rho$-Kraus decomposition, where the desired condition (141) follows from the orthonormality of the $\left.\left|F_{k}\right\rangle\right\rangle \in \mathcal{K} \otimes \mathcal{H}^{*}$ using equation 61 .

If $\mathcal{A}$ is trace-preserving then

$$
1=\operatorname{Tr}_{\mathcal{K H}^{*}} \mathcal{A}\left(\left|\psi_{\rho}\right\rangle_{\mathcal{H H}^{*}}\left\langle\psi_{\rho}\right|\right)=\operatorname{Tr}_{\mathcal{K} \mathcal{H}^{*}} \sum p_{k} E_{k}\left|\psi_{\rho}\right\rangle_{\mathcal{H H}^{*}}\left\langle\psi_{\rho}\right| E_{k}^{\dagger}=\sum p_{k}
$$

by the defining orthonormality condition on the $\left\{E_{k}\right\}$, proving that $\left\{p_{k}\right\}$ is a probability distribution. 
Now suppose that we are given an arbitrary $\rho$-Kraus decomposition (140) and that $\mu \in B^{1}(\operatorname{supp}(\rho))$. Note that since both sides of (144) are linear in $\mu$ we may assume without loss of generality that $\mu$ is positive semidefinite. Then by equations $68,148,146,140$, and cyclicity of the trace

$$
\begin{aligned}
\sum f\left(p_{k}\right) E_{k} \mu E_{k}^{\dagger} & =\operatorname{Tr}_{\mathcal{H}^{*}} \sum f\left(p_{k}\right) E_{k}\left|\psi_{\mu}\right\rangle_{\mathcal{H} \mathcal{H}^{*}}\left\langle\psi_{\mu}\right| E_{k}^{\dagger} \\
& =\operatorname{Tr}_{\mathcal{H}^{*}}\left[\overline{\mu^{1 / 2} \rho^{-1 / 2^{+}}}\left(\sum f\left(p_{k}\right) E_{k}\left|\psi_{\rho}\right\rangle_{\mathcal{H} \mathcal{H}^{*}}\left\langle\psi_{\rho}\right| E_{k}^{\dagger}\right) \overline{\rho^{-1 / 2^{+}} \mu^{1 / 2}}\right] \\
& =\operatorname{Tr}_{\mathcal{H}^{*}}\left[\overline{\mu^{1 / 2} \rho^{-1 / 2^{+}}} f\left(\sum p_{k} E_{k}\left|\psi_{\rho}\right\rangle_{\mathcal{H H}^{*}}\left\langle\psi_{\rho}\right| E_{k}^{\dagger}\right) \overline{\rho^{-1 / 2^{+}} \mu^{1 / 2}}\right] \\
& =\operatorname{Tr}_{\mathcal{H}^{*}}\left[\overline{\rho^{-1 / 2^{+}} \mu \rho^{-1 / 2^{+}}} \times f\left(\mathcal{A}\left(\left|\psi_{\rho}\right\rangle_{\mathcal{H H}^{*}}\left\langle\psi_{\rho}\right|\right)\right)\right],
\end{aligned}
$$

as desired.

\subsection{Quadratic quantum error recovery}

Theorem 44 Let $\mathcal{A}: B^{1}(\mathcal{H}) \rightarrow B^{1}(\mathcal{K})$ be a quantum operation, and let $\rho$ be a density matrix on $\mathcal{H}$. Then one has the following bounds on the optimal entanglement fidelity of recovery

$$
\frac{\Lambda^{2}}{\operatorname{Tr} \mathcal{A}(\rho)} \leq F_{e}\left(\rho, \mathcal{R}^{Q R} \circ \mathcal{A}\right) \leq \sup _{\mathcal{R}_{\mathcal{K} \rightarrow \mathcal{H}}} F_{e}(\rho, \mathcal{R} \circ \mathcal{A}) \leq \Lambda,
$$

where the supremum is over quantum operations $\mathcal{R}: B^{1}(\mathcal{K}) \rightarrow B^{1}(\mathcal{H})$, where

$$
\Lambda=\operatorname{Tr}_{\mathcal{K}} \sqrt{\mathcal{A}^{(2, \rho)}\left(\rho^{2}\right)} \leq \operatorname{Tr} \mathcal{A}(\rho),
$$

where $\mathcal{A}^{(2, \rho)}$ is given by Definition 42 (see also equations 147 and 172, below), and where quadratic recovery operation is given by

$$
\mathcal{R}^{Q R}(v)=\rho\left(\mathcal{A}^{(2, \rho)}\right)^{\dagger}\left(\left(\mathcal{A}^{(2, \rho)}\left(\rho^{2}\right)\right)^{-1 / 2^{+}} v_{\mathcal{K} \rightarrow \mathcal{K}}\left(\mathcal{A}^{(2, \rho)}\left(\rho^{2}\right)\right)^{-1 / 2^{+}}\right) \rho .
$$

Here the adjoint $(\bullet)^{\dagger}$ is from Definition 24.

Remark: In the case that $\mathcal{A}$ is trace-preserving, one may plug the square of the last inequality of (153) into the first inequality (153), giving

$$
\frac{F_{e}\left(\rho, \mathcal{R}^{\mathrm{QR}} \circ \mathcal{A}\right)}{\sup _{\mathcal{R}_{\mathcal{K} \rightarrow \mathcal{H}}} F_{e}(\rho, \mathcal{R} \circ \mathcal{A})} \geq \frac{\Lambda^{2}}{\sup _{\mathcal{R}_{\mathcal{K} \rightarrow \mathcal{H}}} F_{e}(\rho, \mathcal{R} \circ \mathcal{A})} \geq \sup _{\mathcal{R}_{\mathcal{K} \rightarrow \mathcal{H}}} F_{e}(\rho, \mathcal{R} \circ \mathcal{A}) .
$$

In particular, both of the lower bounds of (153) are sufficiently tight to also satisfy the tightness relation (34) of Barnum and Knill [14]. (Note, however, that Barnum and Knill also produce estimates for average entanglement fidelity, under certain commutativity assumptions.) Furthermore, by expressing the bounds $(153)$ in terms of the infidelity $1-F_{e}(\rho, \mathcal{R} \circ \mathcal{A})$ one obtains the fact that $\mathcal{R}^{\mathrm{QR}}$, like $\mathcal{R}^{\mathrm{BK}}$, has an infidelity of recovery within a factor of two of the optimal.

Proof. Let $\mathcal{H}_{\text {in }}$ be a copy of $\mathcal{H}$, let $\left|\psi_{\rho}\right\rangle_{\mathcal{H H}^{*}}$ be the canonical purification (66) of $\rho$, and set

$$
\mu_{\mathcal{K H}^{*}}=\mathcal{A}_{\mathcal{H}_{\text {in }} \rightarrow \mathcal{K}}\left(\left|\psi_{\rho}\right\rangle_{\mathcal{H}_{\text {in }} \mathcal{H}^{*}}\left\langle\psi_{\rho}\right|\right) .
$$

Using the replacements $(\mathcal{H}, \mathcal{K}, \mathcal{L}) \rightarrow\left(\mathcal{H}^{*}, \mathcal{K}, \mathcal{H}\right)$ and $|\phi\rangle_{\mathcal{L H}} \rightarrow\left|\psi_{\rho}\right\rangle_{\mathcal{H} \mathcal{H}^{*}}$, Theorem 39 gives estimates of the form

$$
\frac{\tilde{\Lambda}^{2}}{\operatorname{Tr} \hat{\mu}_{\mathcal{K H}} \mathcal{H}^{*}} \leq F_{e}\left(\rho, \mathcal{R}^{\mathrm{QO}} \circ \mathcal{A}\right) \leq \sup _{\mathcal{R}_{\mathcal{K} \rightarrow \mathcal{H}}} F_{e}(\rho, \mathcal{R} \circ \mathcal{A}) \leq \tilde{\Lambda} .
$$

We claim that $\operatorname{Tr} \hat{\mu}_{\mathcal{K} \mathcal{H}^{*}}=\operatorname{Tr} \mathcal{A}(\rho), \tilde{\Lambda}=\Lambda$, and $\mathcal{R}^{\mathrm{QO}}=\mathcal{R}^{\mathrm{QR}}$. 
First claim: Note that

$$
\begin{aligned}
\hat{\mu}_{\mathcal{K} \mathcal{H}^{*}} & =\Pi_{+}\left(\underset{\mathcal{H}}{\operatorname{Tr}}\left|\psi_{\rho}\right\rangle_{\mathcal{H}_{\mathcal{H}^{*}}}\left\langle\psi_{\rho}\right|\right) \mu_{\mathcal{K} \mathcal{H}^{*}} \Pi_{+}\left(\underset{\mathcal{H}}{\operatorname{Tr}}\left|\psi_{\rho}\right\rangle_{\mathcal{H} \mathcal{H}^{*}}\left\langle\psi_{\rho}\right|\right) \\
& =\Pi_{+}\left(\bar{\rho}_{\mathcal{H}^{*}}\right) \mathcal{A}_{\mathcal{H}_{\mathrm{in}} \rightarrow \mathcal{K}}(|\sqrt{\rho}\rangle\rangle_{\mathcal{H}_{\mathrm{in}} \mathcal{H}^{*}}\langle\langle\sqrt{\rho}|) \Pi_{+}\left(\bar{\rho}_{\mathcal{H}^{*}}\right) \\
& =\mathcal{A}_{\mathcal{H}_{\mathrm{in}} \rightarrow \mathcal{K}}\left(\left|\sqrt{\rho} \Pi_{+}(\rho)\right\rangle_{\mathcal{H}_{\mathrm{in}} \mathcal{H}^{*}}\left\langle\left\langle\sqrt{\rho} \Pi_{+}(\rho)\right|\right)\right. \\
& =\mathcal{A}_{\mathcal{H}_{\mathrm{in}} \rightarrow \mathcal{K}}\left(\left|\psi_{\rho}\right\rangle_{\mathcal{H}_{\mathrm{in}} \mathcal{H}^{*}}\left\langle\psi_{\rho}\right|\right)=\mu_{\mathcal{K} \mathcal{H}^{*}},
\end{aligned}
$$

where the first three equalities used (97) \& (94), (69), and (62). It follows by equation 68 that

$$
\underset{\mathcal{K H}^{*}}{\operatorname{Tr}} \hat{\mu}_{\mathcal{K H}^{*}}=\operatorname{Tr}_{\mathcal{K}} \mathcal{A}_{\mathcal{H}_{\mathrm{in}} \rightarrow \mathcal{K}}\left(\underset{\mathcal{H}^{*}}{\operatorname{Tr}}\left|\psi_{\rho}\right\rangle_{\mathcal{H}_{\text {in }} \mathcal{H}^{*}}\left\langle\psi_{\rho}\right|\right)=\operatorname{Tr} \mathcal{A}(\rho)
$$

as desired.

Second claim: One computes

$$
\begin{aligned}
& \left\langle\left.\psi_{\rho}\right|_{\mathcal{H}^{*}}\left(\hat{\mu}_{\mathcal{K H}^{*}}\right)^{2} \mid \psi_{\rho}\right\rangle_{\mathcal{H} \mathcal{H}^{*}} \\
& =\left\langle\left.\psi_{\rho}\right|_{\mathcal{H}_{\mathcal{H}}^{*}}\left(\mathcal{A}_{\mathcal{H}_{\text {in }} \rightarrow \mathcal{K}}\left(\left|\psi_{\rho}\right\rangle_{\mathcal{H}_{\text {in }} \mathcal{H}^{*}}\left\langle\psi_{\rho}\right|\right)\right)^{2} \mid \psi_{\rho}\right\rangle_{\mathcal{H}^{*}} \\
& =\operatorname{Tr}_{\mathcal{H} \mathcal{H}^{*}}\left[\left(\mathcal{A}_{\mathcal{H}_{\text {in }} \rightarrow \mathcal{K}}\left(\left|\psi_{\rho}\right\rangle_{\mathcal{H}_{\text {in }} \mathcal{H}^{*}}\left\langle\psi_{\rho}\right|\right)\right)^{2}\left|\psi_{\rho}\right\rangle_{\mathcal{H} \mathcal{H}^{*}}\left\langle\psi_{\rho}\right|\right] \\
& =\operatorname{Tr}_{\mathcal{H}^{*}}\left[\left(\mathcal{A}_{\mathcal{H}_{\text {in }} \rightarrow \mathcal{K}}\left(\left|\psi_{\rho}\right\rangle_{\mathcal{H}_{\text {in }} \mathcal{H}^{*}}\left\langle\psi_{\rho}\right|\right)\right)^{2} \bar{\rho}_{\mathcal{H}^{*}}\right] \\
& =\mathcal{A}_{\mathcal{H} \rightarrow \mathcal{K}}^{(2, \rho)}\left(\rho^{2}\right),
\end{aligned}
$$

where $\mathcal{H}_{\text {in }}$ is a copy of $\mathcal{H}$ and where our steps (in sequence) used (161), cyclicity of the trace, (69), and (147). That $\tilde{\Lambda}=\Lambda$ now follows from equation 127 .

Third claim: By equation 120

$$
\mathcal{R}_{\mathcal{K} \rightarrow \mathcal{L}}^{\mathrm{QO}}(v)=\operatorname{Tr}_{\mathcal{K H}^{*}}\left[\left(\hat{\mu}_{\mathcal{K} \mathcal{H}^{*}}\right)^{2}\left(\left(Y^{-1 / 2^{+}} v Y^{-1 / 2^{+}}\right)_{\mathcal{K} \rightarrow \mathcal{K}} \otimes\left|\psi_{\rho}\right\rangle_{\mathcal{H} \mathcal{H}^{*}}\left\langle\psi_{\rho}\right|\right)\right]
$$

where by (121) and (165)

$$
Y_{\mathcal{K} \rightarrow \mathcal{K}}=\mathcal{A}^{(2, \rho)}\left(\rho^{2}\right)
$$

But by (66) \& (62), (147), and (65) one has

$$
\begin{aligned}
& \operatorname{Tr}_{\mathcal{H}^{*}}\left[\left(\hat{\mu}_{\mathcal{K H}^{*}}\right)^{2}\left|\psi_{\rho}\right\rangle_{\mathcal{H} \mathcal{H}^{*}}\left\langle\psi_{\rho}\right|\right] \\
& =\operatorname{Tr}_{\mathcal{H}^{*}}\left[\left(\mathcal{A}_{\mathcal{H}_{\text {in }} \rightarrow \mathcal{K}}\left(\left|\psi_{\rho}\right\rangle_{\mathcal{H}_{\text {in }} \mathcal{H}^{*}}\langle\psi \rho|\right)\right)^{2} \bar{\rho}_{\mathcal{H}^{*}}^{-1 / 2^{+}}|\rho\rangle\right\rangle_{\mathcal{H} \mathcal{H}^{*}}\left\langle\langle\rho| \bar{\rho}_{\mathcal{H}^{*}}^{-1 / 2^{+}}\right] \\
& =\left(\mathcal{A}_{\mathcal{H}_{\text {in }} \rightarrow \mathcal{K}}^{(2, \rho)} \otimes \mathbb{1}_{\mathcal{H}}\right)\left({\underset{B}{B^{2}\left(\mathcal{H}^{*}\right) \rightarrow B^{2}\left(\mathcal{H}_{\text {in }}\right)}}^{\operatorname{PT}}\left(|\rho\rangle_{\mathcal{H} \mathcal{H}^{*}}\langle\langle\rho|)\right)\right. \\
& =\left(\mathcal{A}_{\mathcal{H}_{\text {in }} \rightarrow \mathcal{K}}^{(2, \rho)} \otimes \mathbb{1}_{\mathcal{H}}\right)\left(\rho_{\mathcal{H} \rightarrow \mathcal{H}_{\text {in }}} \otimes \rho_{\mathcal{H}_{\text {in }} \rightarrow \mathcal{H}}\right),
\end{aligned}
$$

where $\mathcal{H}_{\text {in }}$ is a copy of $\mathcal{H}$. So setting

$$
X_{\mathcal{K} \rightarrow \mathcal{K}}=Y^{-1 / 2^{+}} v Y^{-1 / 2^{+}}=\left(\mathcal{A}^{(2, \rho)}\left(\rho^{2}\right)\right)^{-1 / 2^{+}} v\left(\mathcal{A}^{(2, \rho)}\left(\rho^{2}\right)\right)^{-1 / 2^{+}}
$$


and substituting (168) into (166) gives

$$
\begin{aligned}
\tilde{\mathcal{R}}_{\mathcal{K} \rightarrow \mathcal{L}}(v) & =\operatorname{Tr}_{\mathcal{K} \mathcal{H}^{*}}\left(\left(\hat{\mu}_{\mathcal{K} \mathcal{H}^{*}}\right)^{2}\left(X_{\mathcal{K} \rightarrow \mathcal{K}} \otimes\left|\psi_{\rho}\right\rangle_{\mathcal{H H}^{*}}\left\langle\psi_{\rho}\right|\right)\right) \\
& =\operatorname{Tr}_{\mathcal{K}}\left(\operatorname{Tr}_{\mathcal{H}^{*}}\left(\left(\hat{\mu}_{\mathcal{K} \mathcal{H}^{*}}\right)^{2}\left|\psi_{\rho}\right\rangle_{\mathcal{H H}^{*}}\left\langle\psi_{\rho}\right|\right) X_{\mathcal{K} \rightarrow \mathcal{K}}\right) \\
& =\operatorname{Tr}_{\mathcal{K}}\left(\mathcal{A}_{\mathcal{H}_{\text {in }} \rightarrow \mathcal{K}}^{(2, \rho)}\left(\rho_{\mathcal{H} \rightarrow \mathcal{H}_{\text {in }}} \otimes \rho_{\mathcal{H}_{\text {in }} \rightarrow \mathcal{H}}\right) X_{\mathcal{K} \rightarrow \mathcal{K}}\right) \\
& =\operatorname{Tr}_{\mathcal{H}_{\text {in }}}\left[\left(\rho_{\mathcal{H} \rightarrow \mathcal{H}_{\text {in }}} \otimes \rho_{\mathcal{H}_{\text {in }} \rightarrow \mathcal{H}}\right)\left(\left(\mathcal{A}^{(2, \rho)}\right)_{\mathcal{K} \rightarrow \mathcal{H}_{\text {in }}}^{\dagger}(X)\right)\right] \\
& =\rho_{\mathcal{H}_{\text {in }} \rightarrow \mathcal{H}}\left(\left(\mathcal{A}^{(2, \rho)}\right)_{\mathcal{K} \rightarrow \mathcal{H}_{\text {in }}}^{\dagger}(X)\right) \rho_{\mathcal{H} \rightarrow \mathcal{H}_{\text {in }}} .
\end{aligned}
$$

This proves the claim.

The inequality $\Lambda \leq \operatorname{Tr} \mathcal{A}(\rho)$ follows from (153).

The following proposition puts our recovery bounds into a form closer to the nearly simultaneouslyappearing bounds of Bény and Oreshkov (Theorem 21, above):

Proposition 45 Suppose that $\rho$ is a density on $\mathcal{H}$ and that the quantum operation $\mathcal{A}: B^{1}(\mathcal{H}) \rightarrow$ $B^{1}(\mathcal{K})$ has a Kraus decomposition of the form

$$
\mathcal{A}(\mu)=\sum F_{k} \mu F_{k}^{\dagger}
$$

where the $F_{k}$ are not constrained to satisfy any orthogonality conditions. Then for $\mu \in B^{1}(\operatorname{supp}(\rho))$ one has

$$
\mathcal{A}^{(2, \rho)}(\mu)=\sum_{k \ell} F_{k} \mu F_{\ell}^{\dagger} \times \operatorname{Tr}\left(F_{k}^{\dagger} F_{\ell} \rho\right)
$$

Proof. Since both sides of (172) are linear in $\mu$, we may assume without loss of generality that $\mu$ is positive semidefinite. The conclusion follows using equations 147, 148, $66 \& 62$, and $61 \& 64$ (in said order):

$$
\begin{aligned}
\mathcal{A}^{(2, \rho)}(\mu) & =\operatorname{Tr}_{\mathcal{H}^{*}}\left(\overline{\mu^{1 / 2} \rho^{-1 / 2^{+}}} \times\left(\sum_{k} F_{k}\left|\psi_{\rho}\right\rangle_{\mathcal{H} \mathcal{H}^{*}}\left\langle\psi_{\rho}\right| F_{k}^{\dagger}\right)^{2} \times \overline{\rho^{-1 / 2^{+}} \mu^{1 / 2}}\right) \\
& =\operatorname{Tr}_{\mathcal{H}^{*}}\left(\sum_{k \ell} F_{k}\left|\psi_{\mu}\right\rangle_{\mathcal{H} \mathcal{H}^{*}}\left\langle\psi_{\rho}\left|F_{k}^{\dagger} F_{\ell}\right| \psi_{\rho}\right\rangle_{\mathcal{H H}^{*}}\left\langle\psi_{\mu}\right| F_{\ell}^{\dagger}\right) \\
& =\operatorname{Tr}_{\mathcal{H}^{*}}\left(\sum_{k \ell}\left|F_{k} \sqrt{\mu}\right\rangle\right\rangle_{\mathcal{K H}^{*}}\left\langle\left\langle F_{k} \sqrt{\rho}|| F_{\ell} \sqrt{\rho}\right\rangle\right\rangle_{\mathcal{K} \mathcal{H}^{*}}\left\langle\left\langle F_{\ell} \sqrt{\mu}\right|\right) \\
& =\sum_{k \ell} F_{k} \mu F_{\ell}^{\dagger} \times \operatorname{Tr}\left(F_{k}^{\dagger} F_{\ell} \rho\right) .
\end{aligned}
$$

\subsection{The relationship between the Quadratic Recovery and Barnum and Knill's reversal}

As we have already seen in equation 156, the quadratic reversal $\mathcal{R}^{Q R}$ and the simple lower bound of (153) are both sufficiently accurate to also satisfy the tightness relation (34) of Barnum and Knill. This section makes a brief comparison of the quadratic reversal operation with the reversal map of Barnum and Knill (for the special case of non-average entanglement fidelity) in light of the relationship between the quadratic measurement and the PGM. 
Re-expressing the elements of the ensemble (2) as $\rho_{k}=p_{k} \hat{\rho}_{k}$, where $\operatorname{Tr}\left(\hat{\rho}_{k}\right)=1$ and $p_{k}=\operatorname{Tr} \rho_{k}$ is the chance that $\hat{\rho}_{k}$ appears, the formulas for the "pretty good" and quadratically-weighted measurements become

$$
\begin{aligned}
M_{k}^{\mathrm{PGM}} & =\left(\sum p_{\ell} \hat{\rho}_{\ell}\right)^{-1 / 2^{+}} p_{k} \hat{\rho}_{k}\left(\sum p_{\ell} \hat{\rho}_{\ell}\right)^{-1 / 2^{+}} \\
M_{k}^{\mathrm{QW}} & =\left(\sum p_{\ell}^{2} \hat{\rho}_{\ell}^{2}\right)^{-1 / 2^{+}} p_{k}^{2} \hat{\rho}_{k}^{2}\left(\sum p_{\ell}^{2} \hat{\rho}_{\ell}^{2}\right)^{-1 / 2^{+}} .
\end{aligned}
$$

In particular, to get from the pretty-good measurement to the quadratic measurement, one replaces all probabilities and density matrices by their squares.

A simple examination of the formulas (33) and (155) shows that a similar relationship exists between the entanglement fidelity case of the Barnum-Knill reversal $\mathcal{R}^{\mathrm{BK}}$ and the quadraticallyweighted reversal $\mathcal{R}^{\mathrm{QR}}$. Note that the corresponding probabilities $p_{k}$, which must be replaced by their squares, are viewed as being hidden in the $\rho$-Kraus decomposition (140) of the reversed map $\mathcal{A}$.

In [83] various weightings for Belavkin pure-state square-root measurements were compared, and it was argued that Holevo's quadratically-weighted measurement had qualitative and quantitative advantages over the linearly weighted PGM. Based on analogy, we conjecture that $\mathcal{R}^{\mathrm{QR}}$ will typically (but not always) outperform $\mathcal{R}^{\mathrm{BK}}$.

\subsubsection{Depolarizing noise and the quadratic transpose channel}

It is perhaps interesting to quantitatively compare the actions of the Barnum-Knill reversal $\mathcal{R}^{\mathrm{BK}}$ with the quadratic recovery $\mathcal{R}^{\mathrm{QR}}$ in the simplest special case, in which depolarizing noise

$$
\left(\mathcal{A}_{p}\right)_{\mathcal{H} \rightarrow \mathcal{H}}(\mu)=p \times \frac{\mathbb{1}}{\operatorname{dim} \mathcal{H}} \operatorname{Tr}(\mu)+(1-p) \times \mu, \quad p \in[0,1]
$$

acts on half of a maximally-entangled state. (Note that the $\rho=\mathbb{1} / \operatorname{dim} \mathcal{H}$ case of $\mathcal{R}^{\mathrm{BK}}$, also known as the transpose channel [84], has recently [20] been employed in the study of approximate quantum error correction.) For $\operatorname{dim} \mathcal{H}>1$, one easily obtains

$$
\begin{aligned}
& \mathcal{R}_{p}^{\mathrm{BK}}=\mathcal{A}_{p} \\
& \mathcal{R}_{p}^{\mathrm{QR}}=\mathcal{A}_{f(p, \operatorname{dim} \mathcal{H})},
\end{aligned}
$$

where

$$
f(p, \operatorname{dim} \mathcal{H}):=\frac{p^{2}}{(1-p)^{2}(\operatorname{dim} \mathcal{H})^{2}+(2-p) p}
$$

satisfies

$$
f(p, \operatorname{dim} \mathcal{H}) \leq f(p, 1)=p^{2} .
$$

In particular, both recovery operations "correct" depolarization errors by committing further depolarization. Fortunately, however, when $p<1$ the quadratic recovery depolarizes with lower probability than the transpose channel, especially when $\operatorname{dim} \mathcal{H}$ is large or $p$ is small.

A more detailed quantitative comparison of $\mathcal{R}^{\mathrm{BK}}, \mathcal{R}^{\mathrm{QR}}$, and of reversals of other possible weightings (perhaps generalizing the cubically-weighted measurement of [91, 92]) will be left for future work.

\section{Conclusion and future directions}

We have generalized the iterative schemes of Ježek-Řeháček-Fiurášek [74], Ježek-Fiurášek-Hradil $[6,75]$, and Reimpell-Werner [7, 10]. Using an abstract framework, "small angle" guesses were employed to construct concise two-sided bounds for minimum-error quantum detection, maximum overlap, quantum conditional min-entropy, and the reversibility of quantum dynamics. An 
approximately-optimal channel reversal and overlap operation were derived. The resulting bounds were sufficiently tight to also satisfy the tightness relations of Barnum and Knill [14], although our methods more generally allowed the target state and the input state to differ. Our recovery operation was found to be a significant improvement of the transpose channel in the simple case of depolarizing noise acting on half of a maximally-entangled state.

As a direction for future study, we note that Barnum and Knill constructed an approximate reversal operation in the more general sense of average entanglement fidelity, albeit with commutativity assumptions of unknown necessity. A remaining open question is whether one can generalize our quadratic reversal construction to this case of average entanglement fidelity, and whether these commutativity assumptions may be removed. More generally, one may ask how to obtain estimates for the maximum overlap problem without our assumed purity of the target state. The principle difficulty in answering both of these questions is in finding an appropriate "small angle guess," in the sense of lemma 7.

Another future direction, in which we have made recent progress [93], is to employ matrix monotonicity to obtain bounds for the maximum overlap problem, including its special cases of channel reversibility and quantum conditional min-entropy.

\section{Appendix A: Canonical Stinespring dilations}

Using only the square root function and the natural isomorphisms of Section 2.1, one may construct Stinespring dilations which are independent of any choice of a basis:

Definition 46 Let $\mathcal{R}: B^{1}(\mathcal{K}) \rightarrow B^{1}(\mathcal{L})$ be a completely positive map, with $\mathcal{K}$ finite-dimensional. The canonical environment is given by

$$
\mathcal{E}=\mathcal{L}_{\mathcal{E}}^{*} \otimes \mathcal{K}_{\mathcal{E}}
$$

where $\mathcal{L}_{\mathcal{E}}^{*}$ and $\mathcal{K}_{\mathcal{E}}$ are copies of $\mathcal{L}^{*}$ and $\mathcal{K}$, respectively. The canonical Stinespring dilation $U_{\mathcal{R}}$ of $\mathcal{R}$ is the linear transformation $U_{\mathcal{R}}: \mathcal{K} \rightarrow \mathcal{L} \otimes \mathcal{E}$ such that $\left|U_{\mathcal{R}}\right\rangle_{\mathcal{L} \mathcal{E} \mathcal{K}^{*}}=|U\rangle_{\mathcal{L} \mathcal{K}^{*} \mathcal{L}_{\mathcal{E}}^{*} \mathcal{K}_{\mathcal{E}}}$ is the canonical purification (66) of the Choi matrix $\tilde{\mathcal{R}}=\mathcal{R}(|\mathbb{1}\rangle\rangle_{\mathcal{K} \mathcal{K}^{*}}\langle\langle\mathbb{1}|)$.

That $U_{\mathcal{R}}$ is a bona fide purification of $\mathcal{R}$ follows from the following lemma:

Lemma 47 Let $\mathcal{R}: B^{1}(\mathcal{K}) \rightarrow B^{1}(\mathcal{L})$ be a quantum operation, with $\mathcal{K}$ finite-dimensional. Then $U_{\mathcal{K} \rightarrow \mathcal{L E}}$ is a Stinespring dilation of $\mathcal{R}$ iff $\left.|U\rangle\right\rangle_{\mathcal{L E \mathcal { K } ^ { * }}}$ is a purification of the Choi matrix $\tilde{\mathcal{R}}$.

Proof. Suppose that $U$ dilates $\mathcal{R}$. Then by equation 62

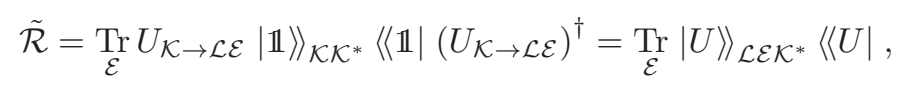

so $|U\rangle\rangle$ purifies $\tilde{\mathcal{R}}$.

Conversely, suppose that $|U\rangle_{\mathcal{L E} \mathcal{K}^{*}}$ purifies $\tilde{\mathcal{R}}$, and let $v \in B^{1}(\mathcal{K})$ be a density matrix. Then by equations $64,62,66$, and 68 ,

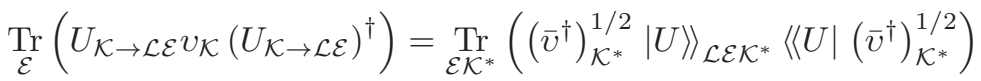

$$
\begin{aligned}
& =\operatorname{Tr}_{\mathcal{K}^{*}}\left[\left(\bar{v}^{\dagger}\right)_{\mathcal{K}^{*}}^{1 / 2} \mathcal{R}(|\mathbb{1}\rangle\rangle_{\mathcal{K} \mathcal{K}^{*}}\langle\langle\mathbb{1}|)\left(\bar{v}^{\dagger}\right)_{\mathcal{K}^{*}}^{1 / 2}\right] \\
& =\mathcal{R}\left(\operatorname{Tr}_{\mathcal{K}^{*}}\left|\psi_{v}\right\rangle\left\langle\psi_{v}\right|\right)=\mathcal{R}(v) \text {. }
\end{aligned}
$$




\section{Appendix B: Reimpell-Werner iteration as directional itera- tion}

The purpose of this section is to verify that Reimpell-Werner iteration (introduced in section 1.4.2) for CP maps corresponds to directional iteration of the corresponding Stinespring dilations.

One may re-express the maximized functional $f(\mathcal{R})$ of equation $(21)$ as

$$
f(\mathcal{R})=\left\|U_{\mathcal{R}}\right\|_{F}^{2},
$$

where $U_{\mathcal{R}}$ is a Stinespring dilation of the CP map $\mathcal{R}: B^{1}(\mathcal{K}) \rightarrow B^{1}(\mathcal{L})$ and the seminorm is defined by

Definition 48 Let $\mathcal{E}=\mathcal{L}_{\mathcal{E}}^{*} \otimes \mathcal{K}_{\mathcal{E}}$ be the canonical environment (178) for quantum operations from $\mathcal{K}$ to $\mathcal{L}$. For operators $U, W: \mathcal{K} \rightarrow \mathcal{L} \otimes \mathcal{E}$, define the semidefinite inner product

$$
\langle U, W\rangle_{F}=\left\langle\left\langle\left. U\right|_{\mathcal{L K} \mathcal{E}^{*}} F_{\mathcal{L} \mathcal{K}^{*} \rightarrow \mathcal{L} \mathcal{K}^{*}} \mid W\right\rangle\right\rangle_{\mathcal{L} \mathcal{K} \mathcal{E}^{*}},
$$

where $A \mapsto|A\rangle\rangle$ is the isomorphism of equation 58. Let $V_{F}=\left\{U \mid\|U\|_{F}<\infty\right\}$, on which $\langle\bullet, \bullet\rangle_{F}$ is a well-defined semidefinite inner product. Let $S=\{U \mid\|U\| \leq 1\}$.

Theorem 49 (Reimpell-Werner iteration is directional iteration) Suppose that $U_{\mathcal{K} \rightarrow \mathcal{L E}}$ is a Stinespring dilation of a CP map $\mathcal{R}: B^{1}(\mathcal{K}) \rightarrow B^{1}(\mathcal{L})$. Then $U \in V_{F}$ has a directional iterate $U^{(+)} \in S$ which dilates the Reimpell-Werner iterate $\mathcal{R}^{\oplus}$ of Def. 11.

Proof. Let $X_{\mathcal{K} \rightarrow \mathcal{L E}}$ be the operator defined by

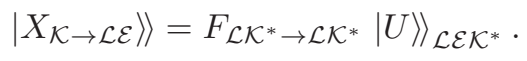

Then by equations 181,43 , and 44 ,

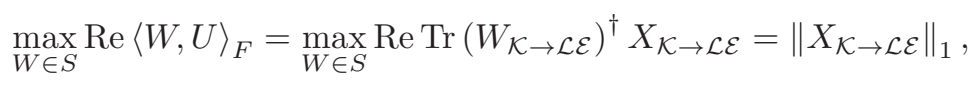

with maximizer $W=U^{(+)}$given by

$$
U^{(+)}=X\left(X^{\dagger} X\right)^{-1 / 2^{+}} .
$$

By equations 62,63 , and 23 one has

$$
\begin{aligned}
\left.\left|U^{(+)}\right\rangle\right\rangle_{\mathcal{L E \mathcal { K } ^ { * }}} & =\left(\overline{X^{\dagger} X}\right)_{\mathcal{K}^{*} \rightarrow \mathcal{K}^{*}}^{-1 / 2^{+}}|X\rangle_{\mathcal{L E} \mathcal{K}^{*}} \\
& =\left(\operatorname{Tr}_{\mathcal{L} \mathcal{E}}|X\rangle_{\mathcal{L E} \mathcal{K}^{*}}\langle\langle X|)^{-1 / 2}|X\rangle\right\rangle_{\mathcal{L E} \mathcal{K}^{*}} \\
& =\left(\operatorname{Tr}_{\mathcal{L} \mathcal{E}} F_{\mathcal{L} \mathcal{K}^{*} \rightarrow \mathcal{L} \mathcal{K}^{*}}|U\rangle_{\mathcal{L} \mathcal{E} \mathcal{K}^{*}}\left\langle\langle U| F_{\mathcal{L} \mathcal{K}^{*} \rightarrow \mathcal{L} \mathcal{K}^{*}}\right)^{-1 / 2^{+}} F_{\mathcal{L} \mathcal{K}^{*} \rightarrow \mathcal{L} \mathcal{K}^{*}}|U\rangle\right\rangle_{\mathcal{L E} \mathcal{K}^{*}} \\
& \left.=\Gamma^{-1 / 2^{+}} F_{\mathcal{L} \mathcal{K}^{*} \rightarrow \mathcal{L} \mathcal{K}^{*}} U_{\mathcal{K} \rightarrow \mathcal{L E}}|\mathbb{1}\rangle\right\rangle_{\mathcal{K} \mathcal{K}^{*}} .
\end{aligned}
$$

It follows from Lemma 47 and Eq. 22 that $U^{(+)}$dilates $\mathcal{R}^{\oplus}$. 


\section{Appendix C: The relationship between overlap bounds and state distinguishability}

As remarked in section 4.2, Theorems 1 and 2 of [70] (see also equations 186-189, below) imply that minimum-error distinguishability of a finite collection of quantum states $\mathcal{E}=\left\{\rho_{k}\right\}_{k=1, \ldots, m}$ may be expressed in terms of restricted maximum overlap:

$$
P_{\text {succ }}\left(M^{\text {opt }}\right)=m \times \max _{\mathcal{R}_{\mathcal{H} \rightarrow\left(\mathbb{C}^{m}\right)^{*}}}\left\langle\phi_{\mathbb{C}^{m}\left(\mathbb{C}^{m}\right)^{*}}\left|\mathcal{R}_{\mathcal{H} \rightarrow\left(\mathbb{C}^{m}\right)^{*}}\left(\mu_{\mathcal{H} \mathbb{C}^{m}}\right)\right| \phi_{\mathbb{C}^{m} \mathbb{C}^{m *}}\right\rangle
$$

Here the vector $\phi \in \mathbb{C}^{m} \otimes\left(\mathbb{C}^{m}\right)^{*}$ is the maximally-mixed state

$$
\left.\left|\phi_{\mathbb{C}^{m}\left(\mathbb{C}^{m}\right)^{*}}\right\rangle=\frac{1}{\sqrt{m}}|\mathbb{1}\rangle\right\rangle_{\mathbb{C}^{m} \mathbb{C}^{m *}}:=\frac{1}{\sqrt{m}} \sum|k\rangle_{\mathbb{C}^{m}}|\bar{k}\rangle_{\mathbb{C}^{m *}},
$$

and $\mu \in B^{1}\left(\mathcal{H} \otimes \mathbb{C}^{m}\right)$ is the "quantum-classical" state

$$
\mu_{\mathcal{H} \otimes \mathbb{C}^{m}}=\sum_{k=1}^{m} \rho_{k} \otimes|k\rangle_{\mathbb{C}^{m}}\langle k|,
$$

where the $\rho_{k}$ are normalized as in Definition 1.

If one applies the overlap bounds of Theorem 39 (or the $s=0$ case of Corollary 40 combined with Eq. 12), one obtains

$$
\begin{aligned}
\left(\operatorname{Tr} \sqrt{\sum \rho_{k}^{2}}\right)^{2} \leq P_{\text {succ }}\left(M^{\mathrm{QW}}\right) \leq P_{\text {succ }}\left(M^{\mathrm{opt}}\right) & \leq \sqrt{m}\left\|\left(\mathcal{R}^{\mathrm{opt}}\right)^{\dagger}(|\phi\rangle\langle\phi|)\right\|_{\infty} \times \operatorname{Tr} \sqrt{\sum \rho_{k}^{2}} \\
& \leq \sqrt{m} \times \operatorname{Tr} \sqrt{\sum \rho_{k}^{2}} .
\end{aligned}
$$

In particular, if one neglects the $\left\|\mathcal{R}^{\dagger}\right\|_{\infty}$ factor in the fourth expression of this estimate then one picks up a spurious factor of $\sqrt{m}$ not appearing in the bounds of Theorem 15. (Weakness of the upper bound is not surprising, since $\phi$ and $\mu$ are generally not "reasonably overlappable.")

In order to show how one may apply the fourth term of the overlap estimate (185), we give another proof of Theorem 15. It is hoped that similar methods may lead to sharper upper in other instances of maximum overlap or conditional min-entropy.

An "overlap proof" of Theorem 15. We restrict consideration to the case $\mathcal{E}=\left\{\rho_{k}\right\}_{k=1, \ldots, m}$. Given a quantum operation $\mathcal{R}_{\mathcal{H} \rightarrow \mathbb{C}^{M}}$ one has the identity

$$
m \times\left\langle\phi_{\mathbb{C}^{m}\left(\mathbb{C}^{m}\right)^{*}}\left|\mathcal{R}_{\mathcal{H} \rightarrow\left(\mathbb{C}^{m}\right)^{*}}\left(\mu_{\mathcal{H} \mathbb{C}^{m}}\right)\right| \phi_{\mathbb{C}^{m} \mathbb{C}^{m *}}\right\rangle=P_{\text {succ }}\left(M^{\mathcal{R}}\right),
$$

where $\mu \in B^{1}\left(\mathcal{H} \otimes \mathbb{C}^{m}\right)$ and $\phi \in \mathbb{C}^{m} \otimes \mathbb{C}^{m *}$ are as in equations (183)-(184) and where the POVM $M^{\mathcal{R}}$ corresponding to the operation $\mathcal{R}$ is given by

$$
M_{k}^{\mathcal{R}}:=(\mathcal{R})_{\left(\mathbb{C}^{m}\right)^{*} \rightarrow \mathcal{H}}^{\dagger}\left(|\bar{k}\rangle_{\mathbb{C}^{m *}}\langle\bar{k}|\right), \quad k=1, \ldots, m .
$$

Since any given POVM $M$ may be expressed in the form of (187) for the quantum operation $\mathcal{R}=\mathcal{R}^{M}$ given by

$$
\mathcal{R}_{\mathcal{H} \rightarrow\left(\mathbb{C}^{m}\right)^{*}}^{M}(\rho):=\sum_{k=1}^{m}|\bar{k}\rangle_{\mathbb{C}^{m *}}\langle\bar{k}| \times \operatorname{Tr}\left(M_{k} \rho\right)
$$

maximization of (186) over operations $\mathcal{R}$ gives the identity (182). Taking $M^{\text {opt }}$ to be some optimal measurement, it follows that a maximizer of the LHS of (186) is given by

$$
\mathcal{R}^{\mathrm{opt}}=\mathcal{R}^{M^{\mathrm{opt}}}
$$


where $M^{\mathrm{opt}}$ is an optimal measurement. One estimates

$$
\left\|\left(\mathcal{R}^{\mathrm{opt}}\right)_{\mathbb{C}^{m *} \rightarrow \mathcal{H}}^{\dagger}\left(|\phi\rangle_{\mathbb{C}^{m} \mathbb{C}^{m *}}\langle\phi|\right)\right\|_{\infty}=\| \frac{1}{m} \sum|k\rangle_{\mathbb{C}^{m}}\langle k| \otimes M_{k}^{\mathrm{opt}} \|_{\infty} \leq \frac{1}{m} .
$$

Applying the bounds (126) to (186) yields the chain of inequalities

$$
\left(\sqrt{\sum \rho_{k}^{2}}\right)^{2} \leq P_{\text {succ }}\left(M^{\mathrm{QW}}\right) \leq P_{\text {succ }}\left(M^{\mathrm{opt}}\right) \leq \sqrt{\sum \rho_{k}^{2}} \leq \sqrt{m} \times \sqrt{\sum \rho_{k}^{2}} .
$$

Acknowledgements: We would like to thank Arthur Jaffe, Peter Shor, and Chris King for their encouragement, Arthur Jaffe for suggesting a change in presentation, Andrew Fletcher, Frédéric Dupuis, Cedric Bény, Ognyan Oreshkov \& Renato Renner for valuable discussions, Stephanie Wehner for pointing out the work of Ogawa and Nagaoka, the anonymous referee for valuable comments, and a previous referee for [22] for pointing out the connection to min-entropy.

Note Added: Private communication from the authors of [18] indicates that they have obtained the quadratic recovery channel by alternative means [19].

\section{References}

[1] A. S. Holevo, "Remarks on optimal measurements," Problems of Information Transmission 10, no.4 317-320 (1974); Translated from Problemy Peredachi Informatsii, 10 no. 4, 51-55 (1974).

[2] H. P. Yuen, R. S. Kennedy, and M. Lax, "Optimum testing of multiple hypotheses in quantum detection theory," IEEE Trans. Inf. Theory, IT-21, 125 (1975).

[3] A. S. Holevo, "Statistical Decision Theory for Quantum Systems," Journal of Multivariate Analysis 3, 337-394 (1973).

[4] S. M. Barnett and S. Croke, "On the conditions for discrimination between quantum states with minimum error," J. Phys. A: Math. Theor. 42062001 (2009); e-print arXiv:0810.1919. Note: The presented argument becomes even shorter if one uses Holevo's [1] simplified conditions for optimal measurements, which are precisely given by the positivity of the operators $\hat{G}_{j}$ of equation (10).

[5] B. Schumacher and M. Westmoreland, "Approximate quantum error correction," Quantum Information Processing 1, 5-12 (2002); arXiv:quant-ph/0112106.

[6] M. Ježek, J. Fiurášek, and Z. Hradil, "Quantum inference of states and processes," Physical Review A 68, 012305 (2003); quant-ph/0210146.

[7] M. Reimpell and R. F. Werner, "Iterative optimization of quantum error correcting codes," Phys. Rev. Lett. 94, 080501 (2005); e-print arXiv:quant-ph/0307138. Note: The authors were unaware of the previous work of [6]. Furthermore, despite much suggestive languange, Reimpell and Werner do NOT prove that their iterates converge when the encoder or decoder is held fixed. (See [10], below.)

[8] A. S. Fletcher, P. W. Shor, and M. Z. Win, "Fletcher Shor Win Optimum quantum error recovery using semidefinite programming," Phys. Rev A 75, 012338 (2007); e-print arXiv: quant-ph/0606035.

[9] M. Reimpell, R. F. Werner, and K. Audenaert, "Comment on 'Optimum quantum error recovery using semidefinite programming," e-print arXiv:quant-ph/0606059. 
[10] M. Reimpell, "Quantum information and convex optimization," Ph.D. Thesis, Braunschweig, Technishe Universität, 2007, urn:nbn:de:gbv:084-17795; http://deposit.ddb.de/cgi-bin/dokserv?idn=988217317. Note: The reported "stability" result of Theorem 3.2.13 is not by itself sufficient to prove that the channel power iteration converges to any limit, even when started in an arbitrarily small neighborhood of the global maximum. For example, the mapping $f(x)=x+x^{3}$ on $\mathbb{R}$ satisfies Reimpell's definition of "stable" about the fixed point $x=0$, since $\left\|f^{\prime}(0)\right\| \leq 1$. Note, however, that the iterative sequence $x, f(x), f(f(x)), \ldots$ diverges unless $x=0$. Furthermore, consideration of iterated rotations of the unit ball in $\mathbb{R}^{2}$ shows that an assumption of compactness is no remedy.

[11] A. S. Fletcher, P.W. Shor, and M. Z. Win, "Channel-Adapted Quantum Error Correction for the Amplitude Damping Channel," IEEE Trans. Inf. Theory, 54 5705-5718 (2008); e-print arXiv:0710.1052.

[12] R. Kosut, A. Shabani, D. Lidar, "Robust quantum error correction via convex optimization," Physical Review Letters 100, 020502 (2008); quant-ph/0703274.

[13] S. Taghavi, R. L. Kosut, and D. A. Lidar, "Channel-Optimized Quantum Error Correction," e-print arXiv:0810.2524 (2008).

[14] H. Barnum and E. Knill, "Reversing quantum dynamics with near-optimal quantum and classical fidelity," J. Math. Phys. 43, 2097 (2002); e-print arXiv: quant-ph/0004088. Note: See [108].

[15] A. S. Fletcher, "Channel-Adapted Quantum Error Correction," Ph. D. Thesis MIT Cambridge, MA 2007; e-print arXiv: 0706.3400.

[16] A. S. Fletcher, P. W. Shor, and M. Z. Win, "Structured near-optimal channel-adapted quantum error correction," Phys Rev A 77, 012320 (2008); e-print arXiv: 0708.3658.

[17] Naoki Yamamoto, Shinji Hara, and Koji Tsumura, "Suboptimal quantum-error-correcting procedure based on semidefinite programming," Physical Review A 71, 022322 (2005); quant-ph/0606105.

[18] C. Bény and O. Oreshkov, "General conditions for approximate quantum error correction and near-optimal recovery channels," Physical Review Letters 104, 120501 (2010); arXiv:0907.5391. Note: The term "fidelity" corresponds to the square root of the fidelity as defined above. The statement "However, we will show that when $\hat{\mathcal{M}}$ is a projection (which is the case for error correction)..." before Corollary 3 should read "... (as may be taken to be the case for error correction by setting $\hat{\mathcal{M}}(\rho)=\sigma \times \operatorname{Tr} \rho$ for some density $\sigma$ in the domain of $\mathcal{M}=\mathrm{Id}) . "$

[19] C. Bény and O. Oreshkov, in preparation.

[20] Hui Khoon Ng and Prabha Mandayam, "A simple approach to approximate quantum error correction based on the transpose channel," E-print arxiv:0909.0931.

[21] R. Renner, "Security of quantum key distribution," PhD Thesis, ETH Zurich (2005); quant-ph/0512258.

[22] Jon Tyson, "Two-sided estimates of minimum-error distinguishability of mixed quantum states via generalized Holevo-Curlander bounds," J. Math. Phys. 50, 032106 (2009); arXiv:0907.2094. Note: The author was unaware that the presented upper bound on distinguishability was a special case of a bound in [23]. This was pointed out in the erratum [94].

[23] T. Ogawa and H. Nagaoka, "Strong converse to the quantum coding theorem," IEEE Transactions on Information Theory 45, 2486-2489 (1999); quant-ph/9808063. 
[24] C. W. Helstrom, Quantum Detection and Estimation Theory (Academic, New York 1976).

[25] P. Hausladen, R. Josza, B. Schumacher, M. Westmoreland, and W. K. Wootters, "Classical information capacity of a quantum channel," Phys Rev A 54, 1869 (1996).

[26] B. Schumacher and M. D. Westmoreland, "Sending classical information via noisy quantum channels," Phys Rev A 56, 131 (1997).

[27] A. S. Holevo, "The capacity of the quantum channel with general signal states," IEEE Trans. Inf. Theory 44, 269 (1998).

[28] L. Ip, "Shor's algorithm is optimal," http://lawrenceip.com/papers/hspsdpabstract.html (2003).

[29] D. Bacon, A. M. Childs, and W. van Dam, "Optimal measurements for the dihedral hidden subgroup problem," Chicago J. of Theoret. Comput. Sci. 2006, (2006); e-print arXiv: quant-ph/0501044.

[30] D. Bacon, A. M. Childs, and W. van Dam, "From optimal measurement to efficient quantum algorithms for the hidden subgroup problem over semidirect product groups," Proceedings of the 46th IEEE Symp. Foundations of Computer Science, (IEEE, Los Alamitos, CA, 2005), pp. $469-478$ (2005).

[31] A. M. Childs and W. van Dam, "Quantum algorithm for a generalized hidden shift problem," Proceedings of the 18th ACM-SIAM Symp. Discrete Algorithms, (Society for Industrial and Applied Mathematics, Philadelphia, PA, 2007), pp. 1225-1234; e-print arXiv:quant-ph/0507190.

[32] M. Hayashi, A. Kawachi, and H. Kobayashi, "Quantum measurements for Hidden Subgroup Problems with Optimal Sample Complexity," Quantum Inform. and Compu. 8, 0345 (2008) ; e-print arXiv:quant-ph/0604174.

[33] C. Moore and A. Russell, "For Distinguishing Hidden Subgroups, the Pretty Good Measurement is as Good as it Gets," Quantum Inform. Compu. 7, 752 (2007); e-print arXiv:quant-ph/0501177.

[34] D. Bacon and T. Decker, "The optimal single-copy measurement for the hidden-subgroup problem," Phys. Rev. A 77, 032335 (2008); e-print arXiv:0706.4478.

[35] J. Radhakrishnan, M. Rötteler, and P. Sen, "Random measurement bases, quantum state distinction and applications to the Hidden Subgroup Problem," Algorithmica 55, 490-516 (2009).

[36] Markus Hunziker, David A. Meyer, Jihun Park, James Pommersheim, and Mitch Rothstein, "The geometry of quantum learning," Quantum Information Processing 9, 321-341 (2010); http://arxiv.org/abs/quant-ph/0309059.

[37] V. P. Belavkin and V. Maslov, "Design of Optimal Dynamic Analyzer: Mathematical Aspects of Wave Pattern Recognition" In Mathematical Aspects of Computer Engineering, edited by V. Maslov, pp. 146-237 (Mir, Moscow 1987); e-print arXiv:quant-ph/0412031. Note: The first two equations on page 40 should be $F_{i}=H_{i}\left(L^{\circ}\right)^{-1 / 2}$ and $M_{i}^{\circ}=\left(L^{\circ}\right)^{+1 / 2} D_{i}^{\circ}\left(L^{\circ}\right)^{+1 / 2}$.

[38] P. Hayden, D. Leung, and G. Smith, "Multiparty data hiding of quantum information," Phys Rev A 71, 062339 (2005); quant-ph/0407152.

[39] A. Montanaro, "On the distinguishability of random quantum states," Commun. Math. Phys. 273, 619 (2007); quant-ph/0607011.

[40] D. Qiu, "Minimum-error discrimination between mixed quantum states," Phys Rev A 77, 012328 (2008); arXiv:0707.3970. 
[41] A. Montanaro, "A lower bound on the probability of error in quantum state discrimination," Proc. IEEE Information Theory Workshop 2008, pp. 378-380; e-print arXiv:0711.2012.

[42] D. Qiu and L. Li, "Minimum-error discrimination of quantum states: Bounds and comparisons," Physical Review A 81, 042329 (2010).

[43] A. S. Kholevo, "On asymptotically optimal hypothesis testing in quantum statistics," Theor. Probab. Appl. 23411 (1978). Note: The displayed equation between (8) and (9) should be $\sum_{j} \pi_{j}\left\|\psi_{j}-e_{j}\right\|^{2}=2\left(1-\operatorname{Re} \operatorname{Tr}\left(U \Pi \Gamma^{1 / 2}\right)\right)$. The line just after equation (9) should read "where $V^{*}=\left|\Pi \Gamma^{1 / 2}\right|\left(\Pi \Gamma^{1 / 2}\right)^{-1} \ldots$ ". The final expression in the paper should be $2\left(1-\operatorname{Tr}\left|\Gamma^{1 / 2} \Pi\right|\right)$.

[44] P. J. Curlander, "Quantum Limitations on Communication Systems," Ph. D. Thesis, MIT Cambridge, MA 1979.

[45] M. A. Nielsen and I. L. Chuang, Quantum computation and Quantum information (Cambridge: Cambridge university press 2000).

[46] Aram Harrow and Andreas Winter, "How many copies are needed for state discrimination," quant-ph/0606131.

[47] O. Morgenstern and J. von Neumann, Theory of Games and Economic Behavior, Princeton, 1944.

[48] D. Aharonov and M. Ben-Or, Proc. 29th Annual ACM Symposium on Theory of Computing, p. 176. (New York, ACM 1997).

[49] A. Kitaev, "Quantum computations: algorithms and error correction," Russian Mathematical Surveys 52, 1191-1249 (1997).

[50] E. Knill, R. Laflamme, W. H. Zurek, "Resilient quantum computation: error models and thresholds," Proceedings of the Royal Society of London, Ser. A 454, 365-384 (1998); quant-ph/9702058.

[51] P. Aliferis, D. Gottesman, and J. Preskill, "Quantum accuracy threshold for concatenated distance-3 code," Quant. Inf. Comput. 6, 97-165 (2006); quant-ph/0504218.

[52] D. Aharanov, A. Kitaev, and J. Preskill, "Fault tolerant quantum computation with longrange correlated noise," Physical Review Letters 96, 050504 (2006).

[53] P. Shor, "Scheme for reducing decoherence in quantum computer memory," Physical Review A 52, R2493 (1995).

[54] A. M. Steane, "Error correcting codes in quantum theory," Physical Review Letters 77, 793797 (1996).

[55] A. R. Calderbank and P. Shor, "Good quantum error-correcting codes exist," Physical Review A 54, 1098-1105.

[56] E. Knill and R. Laflamme, "Theory of quantum error-correcting codes," Physical Review A 55, 900-911 (1997).

[57] C. Bennett, D. DiVincenzo, J. Smolin, and W. Wootters, "Mixed-state entanglement and quantum error correction," Physical Review A 54, 3824-3851 (1996).

[58] R. Laflamme, C. Miquel, J. Paz, W. Zurek, "Perfect Quantum Error Correcting Code," Physical Review Letters 77, 198-201 (1996). 
[59] D. Leung, M. A. Nielsen, I. Chuang, and Y. Yamamoto, "Approximate quantum error correction can lead to better codes," Physical Review A 56, 2567-2573 (1997); quant-ph/9704002.

[60] Claude Crépeau, Daniel Gottesman, and Adam Smith, "Approximate quantum errorcorrecting codes and secret sharing schemes," in Advances in Cryptology - EUROCRYPT 2005, Lecture Notes in Computer Science 3494, pp. 285-301 (2005).

[61] B. Schumacher, "Sending entanglement through noisy quantum channels," Physical Review A 54, 2614-2628 (1996).

[62] D. Kretschmann, D. Schlingemann, and R. Werner, "The information-disturbance tradeoff and the continuity of Stinespring's representation," IEEE transactions on information theory 54 \#4 1708-1717 (2008); quant-ph/0605009. Note: Some ambiguity in the introduction is cleared up by Lemma 2.4 of [95].

[63] C. Schaffner, B. Terhal, and S. Wehner, "Robust cryptography in the noisy-quantum-storage model," Quantum Information and Computation 9, 963-996 (2009); arXiv:0807.1333

[64] R. König, S. Wehner, and J. Wullschleger, "Unconditional security from noisy quantum storage," arXiv:0906.1030.

[65] R. Renner, Extracting classical randomness in a quantum world, IEEE Information Theory Workshop, 2008. DOI: 10.1109/ITW.2008.4578686.

[66] C. Schaffner, "Cryptography in the bounded-quantum-storage model," PhD. Thesis, University of Aarhus (2007); arXiv:0709.0289.

[67] A. Leverrier, E. Karpov, P. Grangier, N. Cerf, "Unconditional security of continuous-variable quantum key distribution," arXiv:0809.2252.

[68] R. König and R. Renner, "Sampling of min-entropy relative to quantum knowledge" (2007) arXiv:0712.4291.

[69] R. Renner, S. Wolf, J. Wullschleger, "The single-serving channel capacity," Proceedings of 2006 IEEE International Symposium on Information Theory (2006), pp. 1424-1427; arXiv:cs/0608018.

[70] R. König, R. Renner, and C. Schaffner, "The operational meaning of min- and max-entropy," IEEE Transactions on Information Theory 55, 4337-4346 (2009); e-print arXiv:0807.1338.

[71] S. Wehner, M. Christandl, and A. Doherty, "A lower bound on the dimension of a quantum system given measured data," Physical Review A 78, 062112 (2008); arxiv.org:0808.3960.

[72] Mario Berta, "Single-shot quantum state merging," Ph. D. Thesis ETH Zurich 2008; e-print arXiv: 0912.4495.

[73] Mario Berta, Matthias Christandl, and Renato Renner, "A conceptually simple proof of the quantum reverse Shannon Theorem," E-Print arxiv:0912.3805 (2009).

[74] M. Ježek, J. Řeháček, and J. Fiurášek, "Finding optimal strategies for minimum-error quantum state discrimination," Phys. Rev. A 65, 060301 (2002); quant-ph/0201109.

[75] Z. Hradil, J. Řeháček, J. Fiurášek, and M. Ježek, "Maximum-Likelihood Methods in Quantum Mechanics," Lecture Notes in Physics 649, pp. 59-112 (2004).

[76] J. Fiurášek, and Z. Hradil, "Maximum-likelihood estimation of quantum processes," Physical Review A 63, 020101(R) (2001).

[77] M. Sacchi, "Maximum-likelihood reconstruction of completely positive maps," Physical Review A 63, 054104 (2001). 
[78] M. Mohseni, A. Rezakhani, and D. Lidar, "Quantum process tomography: resource analysis of diferent strategies," Phys. Rev. A 77, 032322 (2008); quant-ph/0702131.

[79] V. P. Belavkin, "Optimal distinction of non-orthogonal quantum signals," Radio Eng. Electron. Phys., 20, 39 (1975).

[80] V. P. Belavkin, "Optimal multiple quantum statistical hypothesis testing." Stochastics 1, 315 (1975). Note: Inequality 4.3 in the statement of Theorem 5 is backwards.

[81] P. Hausladen, "On the Quantum Mechanical Channel Capacity as a Function of the Density Matrix," B. A. Thesis, Williams College, Williamstown, Massachusetts 1993.

[82] P. Hausladen and W. K. Wootters, "A 'pretty good' measurement for distinguishing quantum states," J Mod Optic 41, 2385 (1994).

[83] J. Tyson, "Error rates of Belavkin weighted quantum measurements and a converse to Holevo's asymptotic optimality Theorem," Physical Review A 79, 032343 (2009); arxiv.org:0907.1884.

[84] M. Ohya and D. Petz, Quantum Entropy and its Use, Texts and Monographs in Physics, Springer Verlag, Berlin (1993).

[85] Ky Fan, "Minimax Theorems," Proceedings of the National Academy of Sciences 39, 42-47 (1953).

[86] M. Reed and B. Simon, Methods of Modern Mathematical Physics I: Functional Analysis (Academic, New York, 1980).

[87] W. Forrest Stinespring, "Positive functions on C-* Algebras," Proceedings of the American Mathematical Society 6, 211-216 (1955).

[88] M. D. Choi, "Completely positive linear maps on complex matrices," Linear Algebra and its Applications 10, 285-290 (1975).

[89] K. Kraus, States, effects, and operations (Springer-Verlag, Berlin, 1983).

[90] P. Arrighi and C. Patricot, "On quantum operations as quantum states," Annals of Physics 311, pp. 26-52 (2004); quant-ph/0307024.

[91] Stephanie Wehner, "Cryptography in a quantum world," PhD Thesis: University of Amsterdam (2008); arxiv:0806.3483.

[92] Manuel A. Ballester, Stephanie Wehner, and Andreas Winter, "State discrimination with post-measurement information," IEEE Transactions on Information Theory 54, 4183-4198; quant-ph/0608014.

[93] J. Tyson, In preparation.

[94] J. Tyson, "Erratum: 'Minimum-error quantum distinguishability bounds from matrix monotone functions: A comment on 'Two-sided estimates of minimum-error distinguishability of mixed quantum states via generalized Holevo-Curlander bounds', 'J. Math. Phys 50, 109902 (2009).

[95] B. Rosgen and J. Watrous, "On the hardness of distinguishing mixed-state quantum computations," Proceedings of the 20th annual IEEE conference on computational complexity, 344-354, June 11-15, 2005; arXiv:cs/0407056.

[96] Yonina C. Eldar, Alexandre Megretski, and George C. Verghese, "Designing Optimal Quantum Detectors Via Semidefinite Programming," IEEE Transactions on Information Theory, Vol 49 \#4, pp. 1007-1012 (2003). Note: The reported implementation appears to have mild numerical innaccuracies in the case of optimal measurement operators which are identically 
zero. In particular, the numerical example reported in equation 40 has the unique exact solution $\mu_{1}=(0,0), \mu_{2}=N_{2}^{-1}((1+\sqrt{5}) / 2,1)$, and $\mu_{3}=N_{3}^{-1}((1-\sqrt{5}) / 2,1)$, where $N_{2,3}$ are normalization factors. (The identity $\left|\mu_{1}\right\rangle\left\langle\mu_{1}\right|=0$ follows from the invertibility of $L-\rho_{1}$.) Furthermore, the results concerning the ranks of optimal measurement operators had already been reported in [80].

[97] C. W. Helstrom, "Bayes-Cost reduction algorithm in quantum hypothesis testing," IEEE Trans. Inf. Theory IT-28, 359-366 (1982). Note: Ref. [74] asserts that the presented algorithm does not always converge to an optimal measurement.

[98] J. Tyson, "Estimates of non-optimality of quantum measurements and a simple iterative method for computing optimal measurements," arXiv:0902.0395. Note: Convergence of this algorithm is quite slow.

[99] J. Benedetto and A. Kebo, "The role of frame force in quantum detection," Journal of Fourier Analysis and Applications 14, 443-474 (2008).

[100] J. Baez and J. Huerta, "The algebra of grand unified theories," Bull. Amer. Math. Soc. 47, 483-552 (2010); E-print arXiv:0904.1556.

[101] H. Barnum, C. Caves, C. Fuchs, R. Josza, and B. Schumacher, "On quantum coding for ensembles of mixed states," J. Phys. A: Math. Gen 34 6767-6785 (2001); quant-ph/0008024.

[102] Andreas Winter, "Extrinsic' and 'Intrinsic' Data in Quantum Measurements: Asymptotic Convex Decomposition of Positive Operator Valued Measures," Communications in Mathematical Physics 244, 157-185 (2004); quant-ph/0109050.

[103] Y. C. Eldar and G. D. Forney, "On quantum detection and the square-root measurement," IEEE Trans. Inf. Theory 47, 858 (2001); e-print arXiv:quant-ph/0005132. Note: The authors were unaware of [43].

[104] A. S. Holevo, "Remarks on the Choi-Jamiolkowski Correspondence in Infinite Dimensions," E-Print arXiv:1004.0196 (2010).

[105] One usually requires that a POVM satisfy $\sum M_{k}=\mathbb{1}$. The relaxed condition $\sum M_{k} \leq \mathbb{1}$ allows the possibility that the POVM may fail to return an result. (Alternatively, one may augment $\left\{M_{k}\right\}$ with the operator $M_{\text {error }}=\mathbb{1}-\sum M_{k}$. The operator $M_{\text {error }}$ could perhaps serve as a useful error flag, i.e. an indication that a state orthogonal to $\operatorname{span}(\mathcal{E})$ has been detected.)

[106] Other numerical methods for computing optimal measurements exist [96-99].

[107] The maximum-overlap problem (1) is equivalent to the one-data-point special case of maximum-likelihood quantum process tomography. The specific iteration is defined by equations 14 through 17 of [6].

[108] Barnum and Knill incorrectly assert on page 2103 of [14] that the asymptotically-optimal measurement (24) introduced by Holevo in [43] is equal to the "pretty good" measurement (29).

[109] Indeed, in Quantum Field Theory it is natural to use dual states $\bar{\psi} \in \mathcal{H}^{*}$ to represent antiparticles. See, for example, [100].

[110] Basis-dependent versions may be found in [101, 102].

[111] See [104] for the infinite-dimensional case. 
[112] Indeed, if $\mathcal{E}=\left\{p_{k}\left|\psi_{k}\right\rangle\left\langle\psi_{k}\right|\right\}$ is an ensemble of linearly-independent pure states $\psi_{k}$ spanning $\mathcal{H}$ then the maximizer $E=G^{(+)}$of $\operatorname{Re}\langle G, E\rangle_{\mathcal{E}}$ is of the form $E_{k}=\left|\psi_{k}\right\rangle\left\langle e_{k}\right|$, with $\left\{e_{k}\right\}$ orthornormal. $^{[114]}$ One may therefore express

$$
\sup _{E} \operatorname{Re}\left(\langle G, E\rangle_{\mathcal{E}}\right)=\sup _{\left\{e_{k}\right\}} \operatorname{Re}\left(\sum p_{k}\left\langle e_{k}, \psi_{k}\right\rangle\right)=1-\inf _{\left\{e_{k}\right\}} C^{\text {Holevo }}\left(\left\{e_{k}\right\}\right),
$$

where optimization is over orthonormal $\left\{e_{k}\right\}$. Here $C^{\text {Holevo }}=\frac{1}{2} \sum p_{k}\left\|\psi_{k}-e_{k}\right\|^{2}$ is the "approximate cost function" which was minimized by Holevo ([43], equation 8) in his construction of $M^{\mathrm{QW}}$ in the case of pure states. (See also $[22,103]$.)

[113] One needs the identity $\operatorname{Tr}_{B} \sqrt{\operatorname{Tr}_{A} \rho_{A B} \rho_{A}^{-1 / 2} \rho_{A B}}=\sqrt{\left\langle\psi_{A B}\left|\rho_{A}^{-1 / 2}\right| \psi_{A B}\right\rangle} \times$ $\operatorname{Tr}_{B} \sqrt{\operatorname{Tr}_{A}\left|\psi_{A B}\right\rangle\left\langle\psi_{A B}\right|}=\sqrt{\operatorname{Tr}_{A} \sqrt{\rho_{A}}} \times \operatorname{Tr}_{B} \sqrt{\rho_{B}}=\left(\operatorname{Tr} \sqrt{\rho_{A}}\right)^{3 / 2}$.

[114] Note that if $\left\{\left|e_{k}\right\rangle\left\langle e_{k}\right|\right\}_{k=1, \ldots, \operatorname{dim} \mathcal{H}}$ is a POVM then the $e_{k}$ are orthonormal. In particular, normality of the $e_{k}$ follows from the inequalities $\left\|e_{k}\right\|^{2} \leq \| \sum\left|e_{\ell}\right\rangle\left\langle e_{\ell}\right| \|_{\infty}=1$ and the identity $\sum\left\|e_{\ell}\right\|^{2}=\operatorname{Tr} \sum\left|e_{\ell}\right\rangle\left\langle e_{\ell}\right|=\operatorname{dim} \mathcal{H}$. Orthogonality then follows from the identity $\left\langle e_{\ell}\left|\left(\sum\left|e_{k}\right\rangle\left\langle e_{k}\right|\right)\right| e_{\ell}\right\rangle=\left\|e_{\ell}\right\|^{2}$. 